\title{
Joint Optimization Framework for Operational Cost Minimization in Green Coverage- Constrained Wireless Networks
}

Ganesh Prasad, Deepak Mishra and Ashraf Hossain

The self-archived postprint version of this journal article is available at Linköping University Institutional Repository (DiVA):

http:// urn.kb.se/ resolve?urn=urn:nbn:se:liu:diva-155770

N.B.: When citing this work, cite the original publication.

Prasad, G., Mishra, D., Hossain, A., (2018), J oint Optimization Framework for Operational Cost Minimization in Green Coverage-Constrained Wireless Networks, IEEE Transactions on Green Communications and Networking, 2(3), 693-706. https:/ / doi.org/ 10.1109/ TGCN.2018.2828092

Original publication available at:

https:/ / doi.org/ 10.1109/TGCN.2018.2828092

Copyright: Institute of Electrical and Electronics Engineers (IEEE)

http:// www.ieee.org/ index.html

(C) 2018 IEEE. Personal use of this material is permitted. However, permission to reprint/ republish this material for advertising or promotional purposes or for creating new collective works for resale or redistribution to servers or lists, or to reuse any copyrighted component of this work in other works must be obtained from the IEEE. 


\title{
Joint Optimization Framework for Operational Cost Minimization in Green Coverage-Constrained Wireless Networks
}

\author{
Ganesh Prasad, Member, IEEE, Deepak Mishra, Member, IEEE, \\ and Ashraf Hossain, Senior Member, IEEE
}

\begin{abstract}
In this work, we investigate the joint optimization of base station (BS) location, its density, and transmit power allocation to minimize the overall network operational cost required to meet an underlying coverage constraint at each user equipment (UE), which is randomly deployed following the binomial point process (BPP). As this joint optimization problem is nonconvex and combinatorial in nature, we propose a non-trivial solution methodology that effectively decouples it into three individual optimization problems. Firstly, by using the distance distribution of the farthest UE from the BS, we present novel insights on optimal BS location in an optimal sectoring type for a given number of BSs. After that we provide a tight approximation for the optimal transmit power allocation to each BS. Lastly, using the latter two results, the optimal number of BSs that minimize the operational cost is obtained. Also, we have investigated both circular and square field deployments. Numerical results validate the analysis and provide practical insights on optimal BS deployment. We observe that the proposed joint optimization framework, that solves the coverage probability versus operational cost tradeoff, can yield a significant reduction of about $65 \%$ in the operational cost as compared to the benchmark fixed allocation scheme.
\end{abstract}

Index Terms-Base station deployment, coverage probability, network operational cost, power allocation, global optimization.

\section{INTRODUCTION AND BACKGROUND}

Today with evolution of various applications based on digital world, the number of UEs and demand of data traffic are increasing exponentially without any compromise in the coverage quality of the UEs. For improvement of the coverage, various works are done on deployment strategy of the BSs. The conventional grid model with all the cells being hexagonal in shape and occupying equal area has been shown to be less tractable in a practical environment [2]. Although, deployment of BSs based on homogeneous Poisson point process (HPPP) and binomial point process (BPP) is more tractable for satisfying the practical aspects [3], [4], deterministic deployment of BSs according to distribution of the UEs is more realistic and has been shown to have better performance [5].

In the modern world, data traffic increases almost a factor 10 every 5 years [6]. This causes a huge increment in infrastructure cost every year for meeting the desired Quality

G. Prasad and A. Hossain are with the Department of Electronics and Communication Engineering, National Institute of Technology, Silchar, India (e-mail: \{gpkeshri, ashraf\} @ece.nits.ac.in).

D. Mishra is with the Department of Electrical Engineering, Linköping University, Linköping 58183, Sweden (e-mail: deepak.mishra@liu.se).

A preliminary five-page conference version [1] of this work was presented at IEEE PIMRC, Montreal, Oct. 2017. of Service (QoS). This increment in infrastructure causes significant increase in power dissipation by $16 \%-20 \%$ per year which consumes 180 billion kWh electricity per year, which is nearly $1 \%$ of the world-wide total energy consumption. These huge consumptions of energy result in carbon dioxide $\left(\mathrm{CO}_{2}\right)$ and other greenhouse gases emission of nearly 130 million tons every year [6]. This has led to an indispensable need for QoS-constrained green network deployment strategies that maximize utility of operational cost in achieving a desired coverage demand of all the users intended to be served.

\section{A. Related Works}

Deployment Models: Currently, most of the literature on deployment of BSs are modeled on the distribution of BSs and UEs by HPPP for practical environment. In [3], the authors show that deployment of BSs and UEs by independent HPPP is more tractable and satisfy the practical aspects than placing the BSs on a grid by conventional methods. A survey on modeling of multi-tier networks have been done in [2] using stochastic geometry, where according to type of the network and Media Access Control (MAC) layers, various point processes like Poisson point process, BPP, hard core point process, and Poisson cluster process and their performances have been discussed. However in [4], it was argued that BPP is more realistic and practical network model as compared to HPPP in terms of distribution of the points and size of the network. In contrast to HPPP, in BPP a known and finite number of UEs are distributed in a field. So for better accuracy, we consider a practical setting where UEs are deployed following a BPP.

Power Allocation: One of the method for reduction of power consumption is to dynamically turn BSs on/off based on the time and spatial distribution of the traffic load. Various methods for deciding the sleeping mode of the BSs are discussed in [7]-[15]. Authors in [7] and [8] considered the switching of the BSs based on the traffic profile whereas in [9], both the traffic profile and density of the BSs are considered for deciding the switching. Authors in [10] proposed a switching-based energy saving algorithm which achieves energy savings up to $80 \%$. However, these works [7]-[10] did not consider any Quality of Service (QoS) constraint to be met while minimizing the energy cost. Authors in [11] discussed about the trade off between energy saving and spectral efficiency due to the switching of BSs, and thereby designed an optimal control mechanism to solve this trade 
off. In [12], both centralized and decentralized BS energy saving schemes are proposed under the constraint of outage probability. Authors in [13] and [14] investigated the impact of sleep operation on the blocking probability and delay, respectively. A survey in [15] gives the state of the art on the proposals for reducing the power consumption at the BSs by implementing sleep operations. Although, the works in [7][15] optimize the BSs densities by efficiently controlling the switching operation, they have not discussed about the joint optimization of transmit power and location of the BSs.

BS Localization: Energy efficient network designs by optimizing the BSs densities without any switching of BSs are studied in [16]-[21]. In [18], an energy efficient network is designed by optimizing the densities of BSs without any QoS constraint whereas in [22], blocking probability is taken as a constraint. The coverage probability variation with BS density is studied in [16] for optimizing the power cost. In [19], the optimal combination of macro BSs and micro BSs is investigated for satisfying a minimum data rate. In [17] it was proved that power consumption can be reduced by finding the smallest set of BSs required for a given data traffic load. In [20] and [21], the per unit area power consumption is minimized by optimizing densities of BSs under the constraint of coverage and data rate. However, these works [16]-[21] did not consider the transmit power optimization at BSs while considering the practical deployment constraints.

Operation Cost Minimization: There have been some recent developments [23]-[26] for improving the operational power cost by optimizing more than one parameter of the network. In [23], this improvement is achieved by optimizing the BS densities, their transmit power, and deployment factor of the BSs. A method for reducing the power consumption by optimizing the transmit power, BSs densities, number of BS antennas, and number of UEs per cell in a network, has been proposed in [24]. Considering the joint optimization of BSs density and transmit power under the coverage constraint, it was shown in [25] that coverage performance of the system converges to a fixed value with energy related deployment factor. Authors in [26] first optimized the location and power allocation at the BSs, and then separately optimized the count and location of the BSs. Yet, the joint optimization of number of BSs, their transmit power, and location has not been investigated while incorporating BPP model for UEs.

\section{B. Motivation and Key Contributions}

Although most of the works considered the optimization of randomly deployed BS densities, it has been noted that the deterministic deployment of BS is more realistic and has better performance [5]. To the best of our knowledge, this is the first work that considers coverage-constrained operational cost minimization by jointly optimizing the number of BSs, their transmit power, and locations while considering a realistic $B P P$ for deployment of UEs. Also, the coverage constraint has been applied to the statistically farthest UE in a cell because each BS takes the responsibility for coverage of all associated UEs. Key contributions of this work are as follows.

- Considering a realistic environment for UEs deployment, we have formulated a coverage constraint joint opti- mization problem for minimization of the operational power cost. Due to its nonconvex and combinatorial nature, a non-trivial solution methodology is proposed that decouples the joint problem into three individual optimization problems and provides an efficient way to obtain the joint optimal solution.

- We consider both circular and square field deployments for operational cost minimization while satisfying an average coverage demand. Joint optimal solutions are obtained in each case and the impact of asymptotically high and moderate densities of UEs on the localization of BSs is also discussed. Further, we discuss the method to derive the distribution of the distance between a BS an its UE for differently shaped cells. This distribution is used to obtain the numerically-validated coverage probability of the farthest UE from its BS inside a cell.

- For minimization of power cost over the network, first we jointly optimize the sectoring type involved in the cells generation and the associated location of the BS inside each cell. Here we have shown that the optimal BS localization is based on the minimization of farthest point Euclidean distance in each cell.

- A tight near-optimal analytical approximation for the optimal power allocation is obtained as a function of the underlying BS deployment. We have shown via numerical investigation that this approximation is very tight under practical system constraints and very tightly matches with the global optimal power allocation for high QoS applications having very high coverage quality demand.

- With both optimal BSs location and transmit power allocation obtained as a function of number $N_{B}$ of BSs, we prove that the resulting single variable operational cost is unimodal in $N_{B}$. Using this property an efficient iterative scheme is presented to obtain the optimal number of BSs that in turn yields the optimal BS localization and transmit power allocation providing the minimized operational cost required to meet the underlying coverage demand of each UE in the network.

- Numerical investigation is carried out to validate the analysis and gain nontrivial insights on the impact of various system parameters on the optimized average coverage quality versus cost incurred trade off. A comparison study of operational cost minimization in the square and circular fields having same area is also carried out. Finally, to corroborate the importance of the proposed joint optimization framework, we present its performance comparison against the benchmark schemes to quantify the achievable reduction in operational cost.

\section{SYSTEM MODEL}

In this section, we first introduce the network topology for deployment of BSs and UEs over the circular field. Next, we present the channel model adopted for downlink communication from BSs to UEs, followed by the power cost model for characterizing the operational cost at BSs. 


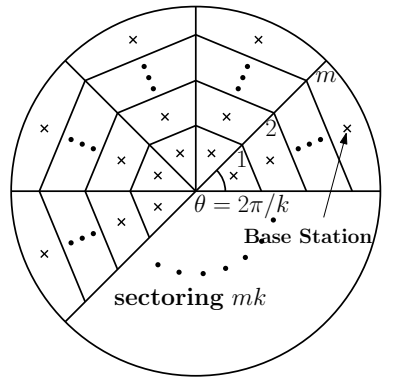

(a)

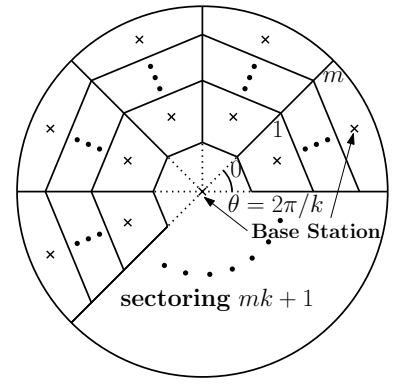

(b)
Fig. 1: Generation of cells in a circular field based on the sectoring (a) $\mathcal{M}: m k=N_{B}$ for $t=0$, (b) $\mathcal{M}: m k+1=N_{B}$ for $t=1$.

\section{A. Network Topology}

We consider a homogeneous cellular network deployment, where $N_{U}$ UEs form a BPP by their independent uniform distribution in a circular field. $N_{B}$ BSs are deterministically deployed over this field for meeting the required average coverage quality for each UE. Under the assumption of mitigation of interference from intracell and intercell downlink communication, the BSs are assumed to employ the orthogonal multi-access techniques [27]. One of the benefit of mitigating the interference is that we are able to find the global optimal solution of the proposed problem with low complexity. Also, it gives the advantage of a noise limited system and highlights the performance and gains of orthogonal systems. The framework comprising the single-input and single-output (SISO) due to its simplicity, low cost antenna requirement with less volume, no processing cost in terms of diversity computation, etc. Downlink association of an UE to a BS in a cell is based on Voronoi-tessellation [28]. Following this, we propose an efficient cell generation method for the circular field to ensure there are no coverage holes and the distance of the farthest point in a cell from its BS is reduced maximally. Here, we first divide the circular field into equal sectors of same angle $\theta$, and then in each sector the BSs are placed along the symmetric line in the radial direction as shown in Fig. 1. Below we define the two sectoring types considered for optimal deployment of BSs over the circular field.

Definition 1: Cells in a circular field are generated by a sectoring $\mathcal{M}: m k+t=N_{B}$ for a given number of deployed BSs $N_{B}$, where the circular field is divided into $k$ equal sectors (each of angle $\theta=2 \pi / k$ ), $m$ BSs are deployed along the symmetric line of each sector and $t=1$ or 0 accounts for a presence or absence of a BS at center of the field, respectively.

All the sectors in sectoring $\mathcal{M}$ have same properties because each of them is generated by dividing the circular field in equal angle $\theta=2 \pi / k$. Therefore, it is sufficient to optimize performance of any one of them. The BSs are deployed along the radial direction in a sector and their locations from the center of the circular field is given by (a) $\mathbf{d}=\left\{d_{i} ; i \in \mathcal{I}\right\}$, where $\mathcal{I}=\{1-t, 2-t, \ldots, m\}$ and $d_{i}$ is the location of the BS in the $i^{t h}$ cell of a sector from the center.

\section{B. Channel Model}

Received power from the BS face a path loss and frequency selective Rayleigh fading. Thus, if the distance of $n^{\text {th }}$ nearest UE from its $\mathrm{BS}$ in the $i^{t h}$ cell is $r_{n, i}$, then the channel power gain is $h_{n, i} r_{n, i}^{-\alpha}$, where $h_{n, i}$ is power of the fading channel coefficient and $\alpha$ is the path loss exponent. $h_{n, i}$ has an exponential distribution as: $h_{n, i} \sim \exp (1)$. The signal-to-noise ratio (SNR) received at that $\mathrm{UE}$ is given by $\gamma_{n, i}=\frac{P_{t} h_{n, i}}{\sigma^{2} r_{n, i}^{\alpha}}$, where $P_{t}$ is the transmit power of each BS and $\sigma^{2}$ is the variance of the zero mean additive white Gaussian noise. The coverage of the $n^{\text {th }}$ nearest UE from the BS depends on whether the received SNR $\gamma_{n, i}$ at that UE is greater than the threshold $T$ required for successfully detecting the information in the received signal. The coverage probability of the $n^{t h}$ nearest UE from the BS at a distance $r_{n, i}$ is $\operatorname{Pr}\left[\gamma_{n, i} \geq T\right]=\operatorname{Pr}\left[h_{n, i} \geq \frac{T \sigma^{2} r_{n, i}^{\alpha}}{P_{t}}\right]=\mathrm{e}^{-\frac{T \sigma^{2} r_{n, i}^{\alpha}}{P_{t}}}$. The coverage probability can be taken as a complementary CDF (CCDF) of $h_{n, i}$ which has an exponential distribution. Using it, the average coverage probability is given as [29, eq. (8)]:

$$
P_{\text {cov }}^{n, i}=\int_{0}^{r_{u, i}} \mathrm{e}^{-\frac{T \sigma^{2} r_{n, i}^{\alpha}}{P_{t}}} f_{n, i}\left(r_{n, i}, \mathbf{d}_{\mathbf{i}}\right) \mathrm{d} r_{n, i}
$$

where $f_{n, i}\left(r_{n, i}, \mathbf{d}_{\mathbf{i}}\right)$ is the probability density function (PDF) of $r_{n, i}$ with $r_{u, i}$ as the upper limit for $r_{n, i}$, and $\mathbf{d}_{\mathbf{i}}=$ $\left\{d_{i-1}, d_{i}, d_{i+1}\right\}$ is the location of the BSs in $(i-1)^{t h}, i^{t h}$, and $(i+1)^{t h}$ cells respectively of a given sector of angle $2 \pi / k$. We notice that the average coverage probability of the $n^{t h}$ nearest UE from the BS in $i^{t h}$ cell depends not only on the location of its own BS, but also on the location of the BSs in adjacent $(i-1)^{t h}$ and $(i+1)^{t h}$ cells, because the boundaries of the cells are determined by the Voronoi diagram. However, in case of $1^{s t}$ and $m^{t h}$ (last) cells, the boundaries along the sector depends only on location of the BSs in $1^{\text {st }}$, $2^{\text {nd }}$ and $(m-1)^{t h}, m^{\text {th }}$ cells, respectively because, one of their boundaries is fixed along the sector. Now if the average coverage probability of the farthest UE from the BS in a cell satisfies a given coverage demand, then statistically it will also be satisfied by the other UEs inside the cell. Hence, in the proposed analysis, we have applied the average coverage constraint only on the farthest UE in a cell.

As our main goal is to minimize the operational cost of the BSs required to meet an average coverage demand, we next present the power cost model for the BS deployment.

\section{Operational Cost Model for the BS Deployment}

From [25], [30], the power consumption model of a BS while doing a downlink transmission is given as:

$$
P_{B S}=N_{P A}\left(\frac{P_{t}}{\mu_{P A}}+P_{S P}\right)\left(1+C_{P C B}\right),
$$

where $P_{B S}$ is the total power consumed by a BS which constitutes of (i) transmit power $P_{t}$ by the BSs, (ii) power amplifier (PA) efficiency $\mu_{P A}$, (iii) total number of power amplifiers $N_{P A}$, (iv) power dissipation $P_{S P}$ in signal processing on the data, and (v) cost $C_{P C B}$ due to power supply, cooling, battery backup and other maintenance costs. This can be further simplified as linear cost model for a BS as: 
$P_{B S}=a_{B} P_{t}+b_{B}$, where $a_{B}$ representing the coefficient of power consumption that scales the radiated power and $b_{B}$ accounting for other consumptions due to signal processing, cooling, and battery backup. Thus, the total operational power cost is $N_{B} P_{B S}$ [25] and it has to be minimized for enabling coverage-constrained green communications.

\section{PRoblem Formulation}

In this section we first obtain average coverage probability of farthest UE as a function of location of the BSs for a given $N_{B}, \mathbf{d}_{\mathbf{i}}, \mathcal{M}$, and the field dimensions. Later, we present the mathematical formulation for the joint optimization problem to minimize the operational cost.

\section{A. Average Coverage Probability of the Farthest UE}

As discussed in Section II-A, we apply the average coverage constraint on the farthest UE inside a cell and it depends on the distribution of the distance $r_{\mathrm{far}, i}$ of the farthest UE. Its $\operatorname{PDF} f_{\text {far }, i}\left(r_{\mathrm{far}, i}, \mathbf{d}_{\mathbf{i}}\right)$ is given by:

$$
\begin{aligned}
f_{\text {far }, i}\left(r_{\text {far }, i}, \mathbf{d}_{\mathbf{i}}\right)= & \sum_{k=0}^{N_{U}}\left(\begin{array}{c}
N_{U} \\
k
\end{array}\right)\left(\frac{A_{i}}{W}\right)^{k}\left(1-\frac{A_{i}}{W}\right)\left(N_{U}-k\right) \\
& \times k f_{i}\left(r_{\mathrm{far}, i}, \mathbf{d}_{\mathbf{i}}\right)\left[F_{i}\left(r_{\mathrm{far}, i}, \mathbf{d}_{\mathbf{i}}\right)\right]^{(k-1)},
\end{aligned}
$$

where $f_{i}\left(r_{\mathrm{far}, i}, \mathbf{d}_{\mathbf{i}}\right)$ is the PDF of $r_{\mathrm{far}, i}$ of an UE in $i^{\text {th }}$ cell, $A_{i}$ is the area of the $i^{t h}$ cell, and $W$ is area of the circular field [4]. If the shape of a cell is polygon, then $F_{i}\left(r_{\mathrm{far}, i}, \mathbf{d}_{\mathbf{i}}\right)$ and $f_{i}\left(r_{\mathrm{far}, i}, \mathbf{d}_{\mathbf{i}}\right)$ can be obtained by the method discussed in [31] and if the boundary of the cell has circular arc, then it can be calculated using appendix A. So, using (1) and (3), the average coverage probability of farthest UE is given by

$$
P_{\operatorname{cov}}^{\mathrm{far}, i}=\int_{0}^{r_{u, i}} \mathrm{e}^{-\frac{T \sigma^{2} r_{\mathrm{far}, i}^{\alpha}}{P_{t}}} f_{\mathrm{far}, i}\left(r_{\mathrm{far}, i}, \mathbf{d}_{\mathbf{i}}\right) \mathrm{d} r_{\mathrm{far}, i} .
$$

From (4), it is evident that average coverage probability of the farthest UE in $i^{t h}$ cell not only depends on the location of its own BS, but also depends on the locations of the BSs in its neighboring $(i-1)^{t h}$ and $(i+1)^{t h}$ cells in the sector.

\section{B. Optimization Formulation}

Below we present the joint optimization problem for finding the number $N_{B}$ of BSs to be deployed along with their transmit power $P_{t}$ and locations $\mathbf{d}$ inside a sector to minimize the operational cost incurred in meeting an average coverage demand at farthest UE in each cell.

$$
\begin{aligned}
& \text { (P0): } \underset{N_{B}, P_{t}, \mathbf{d}}{\operatorname{minimize}} \mathrm{N}_{B}\left[a_{B} P_{t}+b_{B}\right], \quad \text { subject to } \\
& C 1: P_{\mathrm{cov}}^{\mathrm{far}, i} \geq 1-\epsilon, \forall i \in \mathcal{I}, \quad C 2: N_{B}=\left\{1, \ldots, N_{B, \max }\right\},(5) \\
& C 3: 0 \leq P_{t} \leq P_{t, \max }, \quad C 4: 0 \leq d_{i} \leq d_{i, \max }, \forall i \in \mathcal{I} .
\end{aligned}
$$

The constraint $C 1$ ensures that the average coverage probability of the farthest UE is greater than or equal to an acceptable threshold $1-\epsilon$ in each cell. Here $0<\epsilon \ll 1$ is decided based on the acceptable threshold that enables a minimum required coverage probability. The linear box constraints $C 3$ and $C 4$ represent the boundary conditions for $P_{t}$ and $d_{i}$, respectively. Here, $P_{t, \max }$ and $d_{i, \max }$ respectively represent the upper bounds on $P_{t}$ and and location $d_{i}$ of BS in $i^{t h}$ cell. In general,

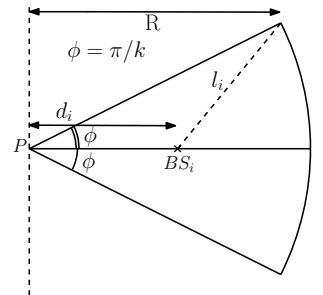

(a) $\mathcal{M}: k=N_{B}, m=1$

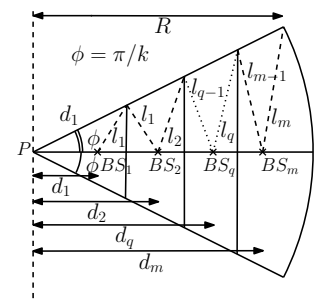

(b) $\mathcal{M}: m k=N_{B}$
Fig. 2: Deployment of cells in a sector of the circular field for $t=0$.

as (P0) is a nonconvex combinatorial optimization problem due to the presence of integer variable $N_{B}$ and non-convexity of the objective function and constraints $C 1$ and $C 2$, it is hard to solve it in its current form. In subsequent sections, we present a nontrivial solution methodology effectively solving this (P0) by decoupling it into three individual problems.

\section{Optimal Deployment Strategy of BSS}

Here we present deployment strategy of BSs over the circular field for a given number $N_{B}$ of BSs. First, we discuss the optimal BSs deployment when number of UEs in the field is asymptotically very high. After that we carry forward the discussion for scenarios with moderate UEs density. Lastly, we demonstrate that optimal deployment strategy of BSs and selection of sectoring type are based on minimizing the farthest point Euclidean distance in each cell.

\section{A. Asymptotically High Density of UEs}

When the number of UEs $N_{U}$ over a finite circular field is asymptotically very high $\left(N_{U} \rightarrow \infty\right)$, then it can be easily shown that in any sub-field of the field, there will be infinite number of UEs, i.e., if $\chi_{i}$ number of UEs is lying in $i^{t h}$ cell (sub-field) of the circular field, then $\chi_{i} \rightarrow \infty$ for $N_{U} \rightarrow \infty$. Therefore, the CDF $F_{\mathrm{far}, i}\left(r_{\mathrm{far}, i}, \mathbf{d}_{\mathbf{i}}\right)$ of distance of farthest UE from location $d_{i}$ of the BS in $i^{\text {th }}$ cell can be expressed as: $F_{\mathrm{far}, i}\left(r_{\mathrm{far}, i}, \mathbf{d}_{\mathbf{i}}\right)=\lim _{\chi_{i} \rightarrow \infty}\left[F_{i}\left(r_{\mathrm{far}, i}, \mathbf{d}_{\mathbf{i}}\right)\right]^{\chi_{i}}$. As CDF $F_{i}\left(r_{\mathrm{far}, i}, \mathbf{d}_{\mathbf{i}}\right)=1$ for $r_{\mathrm{far}, i}=r_{u, i}$ and $<1$ for $r_{\mathrm{far}, i}=r_{u, i}-\nu(\nu>0)$, the corresponding probability of lying of farthest UE over the range $r_{\mathrm{far}, i} \in\left(r_{u, i}-\nu, r_{u, i}\right]$ is:

$$
\begin{gathered}
\operatorname{Pr}\left(r_{u, i}-\nu<r_{\mathrm{far}, i} \leq r_{u, i}\right)=\lim _{\chi_{i} \rightarrow \infty}\left[F_{i}\left(r_{u, i}, \mathbf{d}_{\mathbf{i}}\right)\right]^{\chi_{i}} \\
-\lim _{\chi_{i} \rightarrow \infty}\left[F_{i}\left(r_{u, i}-\nu, \mathbf{d}_{\mathbf{i}}\right)\right]^{\chi_{i}}=1-0=1,
\end{gathered}
$$

where $\nu$ is a very small positive constant $\left(\nu \rightarrow 0^{+}\right)$and $r_{u, i}$ is the farthest point Euclidean distance from the BS. Using (6), PDF of farthest UE can be written as $f_{\mathrm{far}, i}\left(r_{\mathrm{far}, i}, d_{i}\right)=$ $\delta\left(r_{\mathrm{far}, i}-r_{u, i}\right)$ for $\chi \rightarrow \infty$, where $\delta(\cdot)$ is a Dirac delta function. Therefore, if number of UEs in a field is very high, then farthest UE lies at the farthest point Euclidean distance from the BS. So, we determine the optimal location of the BS by minimizing the farthest point Euclidean distance $r_{u, i}$ from it.

The optimal deployment of BSs is based on minimization of UEs' distance from their BSs. It can be attained by minimizing the maximum of the farthest point Euclidean distance over all cells through optimizing the location $\mathbf{d}=\left\{d_{i}\right\}$ of the BSs in a 
sectoring $\mathcal{M}: m k+t=N_{B}$, i.e., $\min _{\mathbf{d}} \max _{i} r_{u, i}, i \in \mathcal{I}$. Here $r_{u, i}$ and $d_{i}$ are the farthest point Euclidean distance and location of the BS respectively in $i^{\text {th }}$ cell. For better understanding, we take an example of sectoring $\mathcal{M}: k=N_{B}(m=1, t=0)$ in which one BS deployed in each sector as shown in Fig. 2(a). In this case $i=1$ which reduces the problem $\min _{\mathbf{d}} \max _{i} r_{u, i}$ to $\min _{d_{i}} r_{u, i}$, where $r_{u, i}=\max \left\{d_{i}, l_{i}\right\}$. For $N_{B} \geq 4, r_{u, i}$ get the minimum value $l_{\min }$ when $d_{i}=l_{i}=\frac{R}{2 \cos \left(\pi / N_{B}\right)}$. Therefore, $\min _{d_{i}} r_{u, i}=l_{\min }=\frac{R}{2 \cos \left(\pi / N_{B}\right)}$ at $d_{i}^{*}=\frac{R}{2 \cos \left(\pi / N_{B}\right)}$, where $l_{i}=\sqrt{d_{i}^{2}+R^{2}-2 d_{i} R \cos \phi}$ for a circular field with radius $R$ and $\phi=\theta / 2=\pi / N_{B}$, for $k=N_{B}$. For $N_{B}=\{1,2\}$, it can be easily shown that $l_{\min }=R$ at $d_{i}^{*}=0$, whereas for $N_{B}=3, l_{\min }=R \sin \phi$ at $d_{i}^{*}=R \cos \phi$ obtained by $\frac{\partial l_{i}^{2}}{\partial d_{i}}=0$.

In general, if we take sectoring $\mathcal{M}: m k=N_{B}$ (for $t=0$ ) as shown in Fig. 2(b), the problem $\min \max r_{u, i}$ reduces to $\min _{\mathbf{d}} \max \left\{d_{1}, l_{1}, l_{2}, \ldots, l_{m}\right\}$, where $l_{i}$ is the Euclidean distance of a vertex from the BS in $i^{t h}$ cell, $r_{u, i}=$ $\max \left\{l_{i-1}, l_{i}\right\} \forall i \in \mathcal{I} \backslash 1$ and $r_{u, i}=\max \left\{d_{i}, l_{i}\right\}$ for $i=1$. Using the similar approach as described for $\mathcal{M}: k=N_{B}$ ( $m=1, t=0$ ), the problem gives the minimum value $l_{\text {min }}$ at $\mathbf{d}^{*}$, when $r_{u, i}=l_{\min }=l_{i}=d_{1} \forall i \in \mathcal{I}$. Therefore, $r_{u, i}=l_{i}=l_{\min } \forall i$ which tells that maximum of the farthest point Euclidean distance over all cells attains a minimum value when farthest point Euclidean distance of all cells becomes equal. Through the trigonometric relationship, the expression of $l_{\text {min }}$ and $d_{i}^{*}$ can be obtained in terms of $N_{B}$ or $k$, where $k$ is related with $N_{B}$ as: $k=\frac{N_{B}-t}{m}$. Likewise, we can also find them in sectoring $\mathcal{M}: m k+1=N_{B}$ (for $t=1$ ). In Table I, the minimized value $l_{\text {min }}$ and corresponding optimal locations $\mathbf{d}^{*}$ are listed in second and third column respectively for different sectoring types (upto sectoring $3 k$ ).

As $\max r_{u, i}$ achieves the minimum value $l_{\min }$ at $\mathbf{d}^{*}$ in

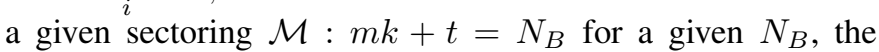
value $l_{\text {min }}$ can be further minimized by optimally choosing a sectoring from a given set of sectoring types. In a set, a sectoring $\mathcal{M}$ becomes optimal when it gives a least value of $l_{\text {min }}$ for a given $N_{B}$. For example, a set of five sectoring $\{k, k+1,2 k, 2 k+1,3 k\}$ obtained by varying $m \in\{1,2,3\}$ and $t \in\{0,1\}$ for $N_{B \text {, max }}=35$ is given in first column of Table $\mathrm{I}$ in which sectoring $k+1$ gives the least value of $l_{\min }$ for $N_{B}=10$. So, sectoring $k+1$ is an optimal sectoring over the set for $N_{B}=10$. The range of $N_{B}$ for which an optimal sectoring gives the least value of $l_{\text {min }}$ has been listed in fourth column of the table. The set of sectoring types is obtained by varying only $m$ and $t$ for a given $N_{B}$ as $k=$ $\frac{N_{B}-t}{m}$ is a dependent variable. The number of elements in a set which is sufficient for obtaining an optimal sectoring $\mathcal{M}^{*}$ for a given $N_{B}$ depends on the maximum number of BSs $N_{B \text {, max }}$ deployed over the field. It can be better understood using the plot in Fig 3 where the minimized value $l_{\min }$ vs. $N_{B}$ is plotted for all the five sectoring types. It can be observed that each sectoring has finite range of $N_{B}$ for which it gives the least value of $l_{\min }$. The sectoring with higher value of $m$ gives the least value of $l_{\min }$ for higher range of $N_{B}$ and vice versa. Therefore, the sectoring with highest value of $m$ in a

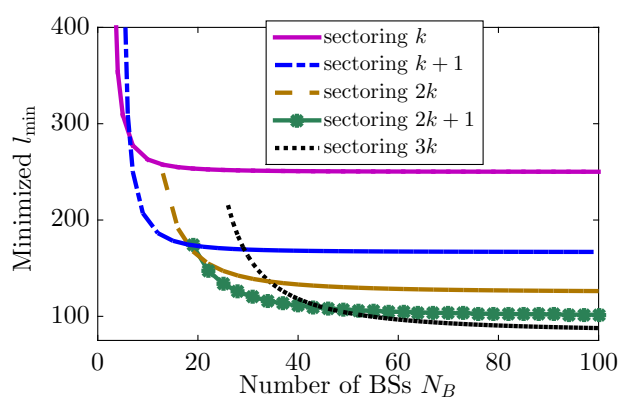

Fig. 3: Variation of minimized $l_{\min }$ with $N_{B}$ for different $\mathcal{M}$.

set is determined by $N_{B \text {,max }}$, i.e., the number of elements in a set is evaluated by $N_{B \text {,max }}$. The range of $N_{B}$ for which a sectoring is optimal can also be evaluated analytically by comparing the expression of $l_{\min }$ of different sectoring types in the set. For example, if we compare the expression of $l_{\min }$ in sectoring $k, k+1$, and $2 k, \frac{R}{4 \cos ^{2}\left(\frac{\pi}{N_{B}-1}\right)-1}<\frac{R}{2 \cos \left(\frac{\pi}{N_{B}}\right)}$ for $N_{B} \geq 4$ and $\frac{R}{4 \cos ^{2}\left(\frac{\pi}{N_{B}-1}\right)-1}<\frac{R}{4 \cos \left(\frac{2 \pi}{N_{B}}\right) \cos \left(\frac{4 \pi}{N_{B}}\right)}$ for $N_{B} \leq 17$ and vice versa. Therefore sectoring $k+1$ has least value of $l_{\min }$ for $4 \leq N_{B} \leq 17$. Similarly, sectoring $2 k$ is optimal for the range $18 \leq N_{B} \leq 19$, but it cannot take the odd integer value, i.e., $N_{B}=19$. So, we have included it in the range for sectoring $k+1$, where $l_{\text {min }}$ has lower value than in the range for sectoring $2 k+1$. Likewise, the range of $N_{B}$ for other sectoring types is evaluated. Thus for a given number of deployed BSs $N_{B}$, the optimal sectoring for deployment of BSs at their optimal location $\mathbf{d}^{*}$ can be evaluated directly using Table I. Although Fig 3 is depicted for a circular field with radius $R=500 \mathrm{~m}$, it is valid for any value of $R$ as it only scales the value of $l_{\text {min }}$ without affecting the range of $N_{B}$ for different sectoring types.

Therefore for a given $N_{B}$, the twofold minimization of maximum of farthest point Euclidean distance over the cells is obtained by optimizing the location of BSs in a sectoring $\mathcal{M}$ as well as the optimization of the sectoring itself from a given set of sectoring types. In other words, the optimization of sectoring minimizes $\max r_{u, i}$ by optimizing the boundaries of the cells through optimal placements of BSs along radial, angular directions, and at the center of the field.

\section{B. When the Density of UEs is Moderate}

Now we investigate the optimal deployment strategy, when the number of UEs $N_{U}$ in the circular field is moderate. Again we consider $f_{\text {far }, i}\left(r_{\mathrm{far}, i}, \mathbf{d}_{\mathbf{i}}\right)$ which is non-zero for $r_{\mathrm{far}, i} \in$ $\left[0, r_{u, i}\right]$. So, it implies that the farthest UE's distance depends on the farthest point Euclidean distance $r_{u, i}$. However, if $r_{u, i}$ gets changed by $\xi>0$ due to a shift in the location of the BS, then there is a non-zero probability for an UE to lie in the distance range $r_{u, i}$ to $r_{u, i}+\xi$ from the BS. But for a given value of $N_{U}$, the farthest UE distance not only depends on $r_{u, i}$, but also on the distribution of area around the boundaries. Therefore, the obtained optimal location $d_{i}^{*}$ based on minimization of farthest point Euclidean distance $r_{u, i}$ is different from actual optimal location $d_{i \text {,act }}^{*}$ which depends on 
TABLE I: $\mathcal{M}^{*}$ and its minimized value $l_{\min }$ of $\max r_{u, i}$ for a given number of BSs $N_{B}$ at their optimal location $\mathbf{d}^{*}$.

\begin{tabular}{|c|c|c|c|}
\hline $\mathcal{M}^{*}$ & Minimized value $l_{\min }$ of $\max _{i} r_{u, i}$ & Optimum location $\mathrm{d}^{*}$ of the BSs & Range of $N_{B}$ \\
\hline \multirow[t]{2}{*}{$k$} & $R \sin \left(\frac{\pi}{N_{B}}\right)$ & $d_{1}^{*}=R \cos \left(\frac{\pi}{N_{B}}\right)$ & $N_{B}=3$ \\
\hline & $\frac{\hat{R}}{2 \cos \left(\frac{\pi}{N_{B}}\right)}$ & $d_{1}^{*}=\frac{\hat{R}}{2 \cos \left(\frac{\pi}{N_{B}}\right)}$ & $N_{B} \in\{4,5,6\}$ \\
\hline$k+1$ & $\frac{R}{4 \cos ^{2}\left(\frac{\pi}{N_{B}-1}\right)-1}$ & $d_{0}^{*}=0, d_{1}^{*}=\frac{2 R \cos \left(\frac{\pi}{N_{B}-1}\right)}{4 \cos ^{2}\left(\frac{\pi}{N_{B}-1}\right)-1}$ & $N_{B} \in\{7,8, \ldots, 17\} \cup\{19\}$ \\
\hline $2 k$ & $\frac{R}{4 \cos \left(\frac{2 \pi}{N_{B}}\right) \cos \left(\frac{4 \pi}{N_{B}}\right)}$ & $\begin{aligned} d_{1}^{*} & =\frac{R}{4 \cos \left(\frac{2 \pi}{N_{B}}\right) \cos \left(\frac{4 \pi}{N_{B}}\right)} \\
d_{2}^{*} & =\frac{R\left(1+\cos \left(\frac{4 \pi}{N_{B}}\right)\right)}{4 \cos \left(\frac{2 \pi}{N_{B}}\right) \cos \left(\frac{4 \pi}{N_{B}}\right)}\end{aligned}$ & $N_{B} \in\{18,20, \ldots, 44\}$ \\
\hline $2 k+1$ & $\frac{R\left(1+2 \cos \left(\frac{4 \pi}{N_{B}-1}\right)\right)}{16 \cos ^{2}\left(\frac{2 \pi}{N_{B}-1}\right) \cos ^{2}\left(\frac{4 \pi}{N_{B}-1}\right)-1}$ & $\begin{array}{c}d_{0}^{*}=0, d_{1}^{*}=\frac{2 R\left(1+2 \cos \left(\frac{4 \pi}{N_{B}-1}\right)\right) \cos \left(\frac{2 \pi}{N_{B}-1}\right)}{16 \cos ^{2}\left(\frac{2 \pi}{N_{B}-1}\right) \cos ^{2}\left(\frac{4 \pi}{N_{B}-1}\right)-1}, \\
d_{2}^{*}=\frac{4 R\left(1+2 \cos \left(\frac{4 \pi}{N_{B}-1}\right)\right) \cos \left(\frac{4 \pi}{N_{B}-1}\right) \cos \left(\frac{2 \pi}{N_{B}-1}\right)}{16 \cos ^{2}\left(\frac{2 \pi}{N_{B}-1}\right) \cos ^{2}\left(\frac{4 \pi}{N_{B}-1}\right)-1}\end{array}$ & $N_{B} \in\{21,23, \ldots, 45\}$ \\
\hline $3 k$ & $\frac{R \cos \left(\frac{3 \pi}{N_{B}}\right)}{\left(2 \cos \left(\frac{6 \pi}{N_{B}}\right)+1\right)\left(\cos \left(\frac{12 \pi}{N_{B}}\right)+\cos \left(\frac{6 \pi}{N_{B}}\right)\right)}$ & $\begin{array}{l}d_{1}^{*}=\frac{R \cos \left(\frac{3 \pi}{N_{B}}\right)}{\left(2 \cos \left(\frac{6 \pi}{N_{B}}\right)+1\right)\left(\cos \left(\frac{12 \pi}{N_{B}}\right)+\cos \left(\frac{6 \pi}{N_{B}}\right)\right)} \\
d_{2}^{*}=\frac{R \cos \left(\frac{3 \pi}{N_{B}}\right)\left(1+2 \cos \left(\frac{6 \pi}{N_{B}}\right)\right)}{\left(2 \cos \left(\frac{6 \pi}{N_{B}}\right)+1\right)\left(\cos \left(\frac{12 \pi}{N_{B}}\right)+\cos \left(\frac{6 \pi}{N_{B}}\right)\right)} \\
d_{3}^{*}=\frac{R \cos \left(\frac{3 \pi}{N_{B}}\right)\left(1+2 \cos \left(\frac{6 \pi}{N_{B}}\right)+2 \cos \left(\frac{9 \pi}{N_{B}}\right)\right)}{\left(2 \cos \left(\frac{6 \pi}{N_{B}}\right)+1\right)\left(\cos \left(\frac{12 \pi}{N_{B}}\right)+\cos \left(\frac{6 \pi}{N_{B}}\right)\right)}\end{array}$ & $N_{B} \in\{48,51, \ldots\}$ \\
\hline
\end{tabular}

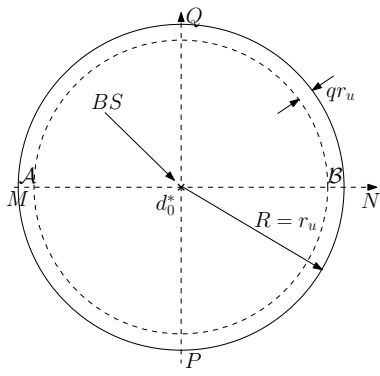

(a) Circular field

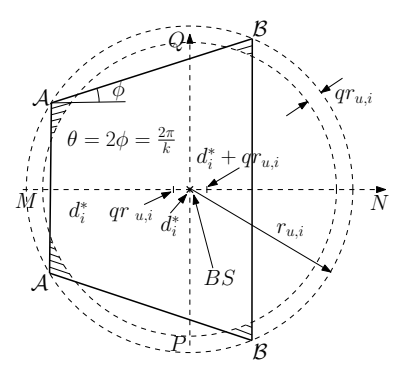

(b) $i^{\text {th }}$ cell for a given $N_{U}$ value
Fig. 4: Depicting the actual optimal location of a BS.

$r_{u, i}$ as well as the distribution of area around the boundaries. To get an insight, we take a scenario when a single BS is deployed at the center of the circular field (cf. Fig. 4(a)). $d_{i \text {,act }}^{*}$ from which the farthest UE's distance is minimum is evaluated by computing its region around $d_{0}^{*}$. The region associated with $d_{0, \text { act }}^{*}$ converges to the optimal location $d_{0}^{*}$ with increment in $N_{U}$ and coincides with it, when $N_{U} \rightarrow \infty$.

For computation of the region, first we discuss the distribution of an area around the boundaries where farthest UE is lying with a probability $1-\psi$ (here $\psi \in(0,1)$ ). Probability of lying of a farthest UE in the range $r_{f a r} \in\left[r_{u}(1-q), r_{u}\right]$ from $d_{0}^{*}$ is given as: $\left[F\left(r_{u}, \mathbf{d}_{\mathbf{0}}^{*}\right)\right]^{N_{U}}-\left[F\left(r_{u}(1-q), \mathbf{d}_{\mathbf{0}}^{*}\right)\right]^{N_{U}} \geq 1-\psi$, where $F\left(r_{\text {far }}, \mathbf{d}_{\mathbf{0}}^{*}\right),\left[F\left(r_{\text {far }}, \mathbf{d}_{\mathbf{0}}^{*}\right)\right]^{N_{U}}$ are CDF of distance $r_{\text {far }}$ of an UE, farthest UE respectively, $\mathbf{d}_{\mathbf{0}}^{*}=\left\{d_{0}^{*}\right\}$, and $r_{u}$ is farthest point Euclidean distance from the BS. As CDF $F\left(r_{\text {far }}, \mathbf{d}_{\mathbf{0}}^{*}\right)=\frac{r_{\text {far }}^{2}}{r_{u}^{2}}$ and $F\left(r_{u}, \mathbf{d}_{\mathbf{0}}^{*}\right)=1$, we get $q \geq 1-\psi^{\frac{1}{2 N_{U}}}$ and for $N_{U} \rightarrow \infty \Rightarrow q \rightarrow 0$ which infers that the farthest UE's distance is same as $r_{u}$ and $d_{0 \text {, act }}^{*}$ coincides with $d_{0}^{*}$. As larger area near to boundaries over the width $q r_{u}$ gives higher probability of lying of a farthest UE in the area, the region of $d_{0, \text { act }}^{*}$ around $d_{0}^{*}$ is nearer to the area for attaining a minimum farthest UE's distance. For its evaluation, two orthogonal axis
$\mathrm{MN}$ and PQ which measures the distribution of area around the boundaries and the region of $d_{0 \text {,act }}^{*}$ around the origin $d_{0}^{*}$ (cf. Fig. 4(a)). The region of $d_{0, \text { act }}^{*}$ on $\mathrm{MN}$ axis is determined by splitting the field about the orthogonal axis PQ followed by the areas $\mathcal{A}$ and $\mathcal{B}$ are compared over the width $q r_{u}$ on the two sides. If $\mathcal{A}>\mathcal{B}$, then probability of lying of farthest $\mathrm{UE}$ on left side of PQ is more than right side. Therefore, the region of $d_{0, \text { act }}^{*}$ is on left side along $\mathrm{MN}$ axis. Similarly, for $\mathcal{A}<\mathcal{B}$, the region is on right side. Due to symmetry of the circular field about the origin $d_{0}^{*}, \mathcal{A}=\mathcal{B}$ which results in no region around $d_{0}^{*}$ along the $\mathrm{MN}$ axis. Likewise the region can be examined along PQ axis after splitting the field about the $\mathrm{MN}$ axis which gives no region again due to symmetry property of the circular field in all directions. Therefore, $d_{0 \text {, act }}^{*}$ exactly coincides with $d_{0}^{*}$ for any value of $N_{U}$.

In general, if we take an $i^{\text {th }}$ cell, when multiple BSs are deployed as shown in Fig. 4(b), the value of $q$ can be obtained numerically by $\sum_{0}^{N_{U}}\left(\begin{array}{c}N_{U} \\ k\end{array}\right)\left(\frac{A_{i}}{W}\right)^{k}\left(1-\frac{A_{i}}{W}\right)^{N_{U}-k}\left(F_{i}((1-\right.$ q) $\left.\left.r_{u, i}, \mathbf{d}_{\mathbf{i}}^{*}\right)\right)^{k} \leq \psi$ which is obtained using (3), where $d_{i}^{*}$ is obtained by minimizing the farthest point Euclidean distance $r_{u, i}$ from the BS in the $i^{t h}$ cell. As the field is symmetric about the MN axis and asymmetric about the orthogonal PQ axis, the region of $d_{i, \text { act }}^{*}$ around $d_{i}^{*}$ is along the MN axis due to the difference in areas $2 \mathcal{A}$ and $2 \mathcal{B}$ on the two sides, where $\mathcal{A}$ and $\mathcal{B}$ are the areas of the field over the width $q r_{u, i}$ near to boundaries as shown in Fig. 4(b) and $d_{i \text {,act }}^{*}$ is actual optimal location of the BS. If $2 \mathcal{A}>2 \mathcal{B}$, then the region on left side over the range $d_{i, \text { act }}^{*} \in\left(d_{i}^{*}-q r_{u, i}, d_{i}^{*}\right)$, for $2 \mathcal{A}<2 \mathcal{B}$, it is on right side over the range $d_{i, \text { act }}^{*} \in\left(d_{i}^{*}, d_{i}^{*}+q r_{u, i}\right)$ and $d_{i \text {,act }}^{*}=d_{i}^{*}$ for $2 \mathcal{A}=2 \mathcal{B}$. Now, we will do a brief discussion on dependence of $d_{i \text {,act }}^{*}$ on $N_{U}$ and number of angular sectors $k$. As discoursed before, with increment in $N_{U}, d_{i \text {,act }}^{*}$ converges to $d_{i}^{*}$ and asymptotically coincides with it, when $N_{U} \rightarrow \infty$. The angle of a sector is $\theta=2 \phi=\frac{2 \pi}{k}$, therefore with increment in $k, \phi$ decreases which results in decrement in difference 
between heights of the rhombus as shown in Figs. 2(b) and 4 (b). Therefore, the difference between the areas $2 \mathcal{A}$ and $2 \mathcal{B}$ decreases and ideally both are equal, when $\phi=0$. So, with increment in $k, d_{i \text {,act }}^{*}$ converges to $d_{i}^{*}$ due to equal area over the width $q r_{u, i}$ on left and right side. Via extensive simulations, we observe that there is diminishable root mean square error (RMSE), when the BSs are deployed based on minimization of farthest point Euclidean distance $d_{i}^{*}$ instead of actual optimal location $d_{i \text {,act }}^{*}$ based on minimization of farthest UE distance which has been shown in Section VII at an instance scenario. Therefore, in the following optimization technique, we will take optimal location of BSs based on minimization of farthest point Euclidean distance for simplicity in calculation.

As optimal location of BSs in a cell is based on minimum value of farthest point Euclidean distance, selection of optimal sectoring $\mathcal{M}^{*}$ is also based on minimization of maximum value of farthest point Euclidean distance over all cells for a given value of $N_{B}$ as described before in Section IV-A.

\section{Proposed Solution Methodology}

Continuing with our solution methodology of solving (P0) by decoupling it into three individual optimization problems, in this section we find the optimal transmit power $P_{t}^{*}$ of BSs and their optimal count $N_{B}^{*}$. With optimal location obtained for a given number $N_{B}$ of BSs in Section IV, now we propose a tight approximation for optimal $P_{t}$ as a function of optimal BS location $\mathbf{d}^{*}$ and optimal sectoring $\mathcal{M}^{*}$ for a given number $N_{B}$ of BSs. Lastly using it, we discuss the reduction of (P0) to a unimodal single variable optimization problem in $N_{B}$ that can be solved efficiently to obtain optimal number $N_{B}^{*}$ of BSs, which will eventually give optimal localization $\left(\mathbf{d}^{*}, \mathcal{M}^{*}\right)$ and transmit power $P_{t}^{*}$ for $N_{B}^{*}$ BSs.

\section{A. Tight Approximation for Optimal Power Allocation}

High QoS applications require very high average coverage probability, i.e., threshold $\epsilon$ is generally very low in practice. Considering this requirement, from constraint $C 1$, we note that to ensure an average coverage of atleast $90 \%$ (i.e., $\epsilon \leq 0.1$ ) for any distribution $f_{\mathrm{far}, i}\left(r_{\mathrm{far}, i}, \mathbf{d}_{\mathbf{i}}\right)$ of farthest UE's distance from its BS in $i^{t h}$ cell, the argument $\frac{T \sigma^{2} r_{\mathrm{far}, i}^{\alpha}}{P_{t}}$ of the exponential term should be $\leq 0.1$. As, $\mathrm{e}^{-x} \approx 1-x, \forall, x \leq 0.1$ with a percentage error $\leq 0.053 \%$ and this approximation error decreases at an exponential rate with decreasing $x$. Applying this approximation to the average coverage probability, constraint $C 1$ in (P0) can be rewritten as

$$
P_{\mathrm{cov}}^{\mathrm{far}, i} \approx 1-\frac{T \sigma^{2}}{P_{t}} \int_{0}^{r_{u, i}} r_{\mathrm{far}, i}^{\alpha} f_{\mathrm{far}, i}\left(r_{\mathrm{far}, i}, \mathbf{d}_{\mathbf{i}}\right) \mathrm{d} r_{\mathrm{far}, i} \geq 1-\epsilon .
$$

Here also, the approximation error is $<0.053 \%$ and it reduces exponentially with decrease in $\epsilon$; it reduces to zero, when the coverage requirement is $100 \%$, i.e., $\epsilon=0$.

Employing this exponential approximation to obtain a tight approximation for optimal power allocation $P_{t}^{*}$ at each BS located at $d_{i}^{*}$, constraint $C 1$ can be rewritten as:

$$
P_{t} \geq \frac{T \sigma^{2}}{\epsilon} \int_{0}^{r_{u, i}} r_{\mathrm{far}, i}^{\alpha} f_{\mathrm{far}, i}\left(r_{\mathrm{far}, i}, \mathbf{d}_{\mathbf{i}}^{*}\right) \mathrm{d} r_{\mathrm{far}, i},
$$

where $\mathbf{d}_{\mathbf{i}}^{*}=\left\{d_{i-1}^{*}, d_{i}^{*}, d_{i+1}^{*}\right\}$ are the optimal location of BSs in $(i-1)^{t h}, i^{t h}$, and $(i+1)^{t h}$ cells, respectively. Since, the operational cost to be minimized is directly proportional to the transmit power $P_{t}$, we need to allocate just sufficient transmit power that can help in achieving the desired coverage threshold $1-\epsilon$. With homogeneous network consideration, the power allocation for all BSs is same and it is obtained by taking the maximum of the power allocations as obtained by solving (8) at strict equality for each BS. Hence, the tight approximation of optimal power allocation is given by

$$
P_{t}^{*} \approx \max _{i}\left\{\frac{T \sigma^{2}}{\epsilon} \int_{0}^{r_{u, i}} r_{\mathrm{far}, i}^{\alpha} f_{\mathrm{far}, i}\left(r_{\mathrm{far}, i}, \mathbf{d}_{\mathbf{i}}^{*}\right) \mathrm{d} r_{\mathrm{far}, i}\right\}
$$

which is a function of number of BSs, $\mathbf{d}_{\mathbf{i}}^{*}$, and $\epsilon$. With transmit power for each BS set as $P_{t}^{*}$ defined in (9), constraint $C 1$ is implicitly satisfied and the value of optimal $P_{t}^{*}$ can be obtained by optimizing the locations $\mathbf{d}^{*}$ of the BSs and corresponding sectoring type $\mathcal{M}^{*}$ for a given value of $N_{B}$.

\section{B. Efficient Iterative Scheme to Find Optimal Number of BSs}

From the developments in Sections IV and V-A, we note that both optimal BS location along with the corresponding optimal sectoring type and transmit power allocation can be represented as a function of $N_{B}$. This reduces the multivariable constrained joint optimization problem (P0) to a univariate problem in $N_{B}$, where $N_{B}$ is a positive integer variable to be optimized. Next, we show that this reduced problem possesses the global optimality property in $N_{B}$.

As with increasing $N_{B}$ the area of the cell to be covered under a BS approximately reduces by a factor of $\frac{1}{N_{B}}$, the resulting distance of the farthest UE from the BS and hence, the transmit power $P_{t}$ required to meet the underlying coverage constraint also decrease by a factor of $\frac{1}{N_{B}^{\beta}}$ where $\beta>0$. Further, the objective function of (P0) is a product of $N_{B}$ and a affine transformation $a_{B} P_{t}+b_{B}$ of $P_{t}$. So on relaxing the integer constraint on $N_{B}$, we note that $N_{B}$ is a positive linear function and $P_{t}$ for a given $N_{B}$ with optimized BS location, as discussed above, is a nonlinear decreasing convex function of $N_{B}$ because the rate of decrease in $P_{t}$ is decreasing with increased $N_{B}$. Using these results in [32, Table 5.2 and Prop. 3.8], the unimodality of the objective in $N_{B}$ is proved. The method for determining the optimal number of BSs $N_{B}^{*}$ and corresponding minimum value of the operational cost is outlined in Algorithms 1 and 2.

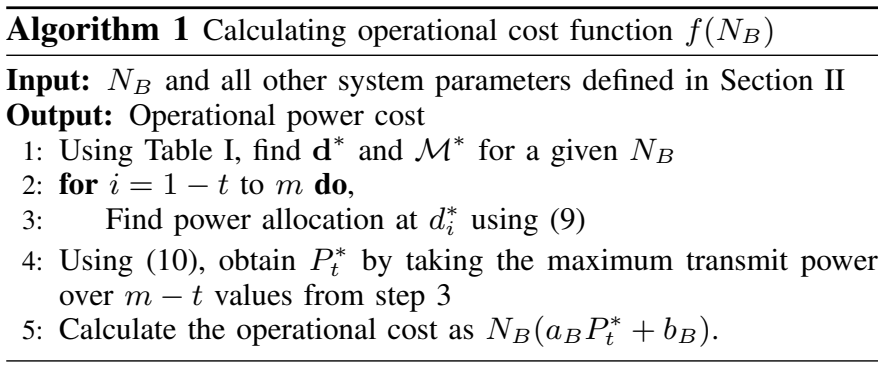

Algorithm 1 outlines a procedure to obtain operational cost as a function $f\left(N_{B}\right)$ of the number $N_{B}$ of BS deployed at optimal locations $\mathbf{d}^{*}$ with optimal sectoring type $\mathcal{M}^{*}$ and power allocation $P_{t}^{*}$. Algorithm 1 starts with the calculation of optimal location $\mathbf{d}^{*}$ of BSs and sectoring $\mathcal{M}^{*}$ for a given 


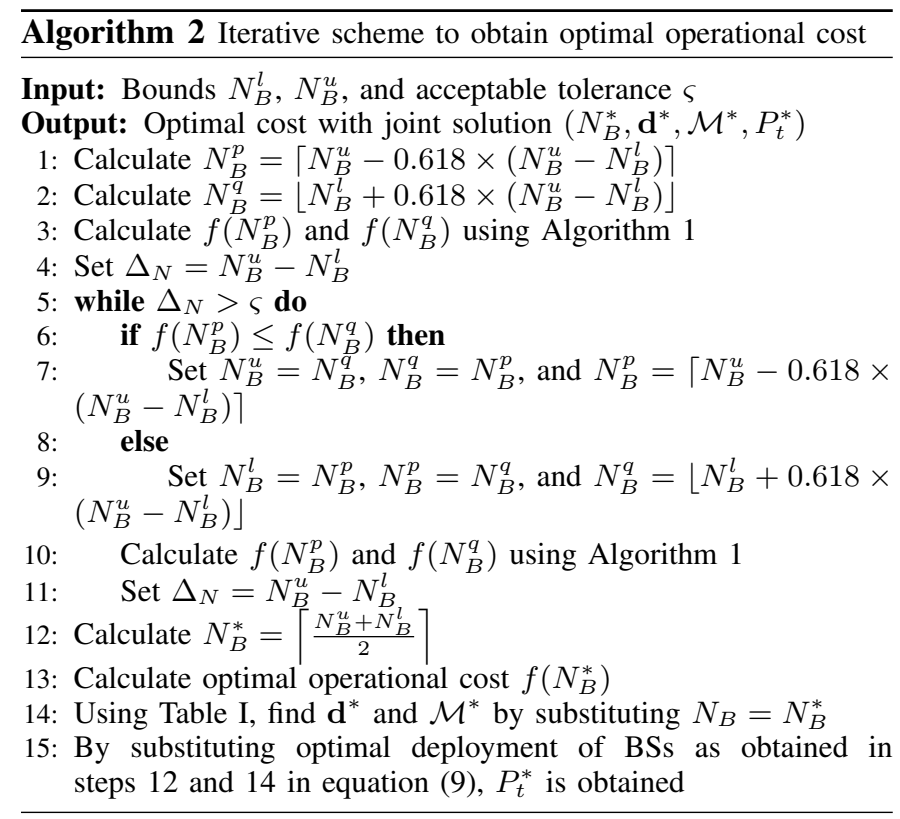

value of $N_{B}$, and then determines the optimal power allocation $P_{t}^{*}$ at $\mathbf{d}^{*}$ in sectoring $\mathcal{M}^{*}$. Finally, it returns the operational cost $f\left(N_{B}\right)$ at $P_{t}^{*}$ for a given value of $N_{B}$. Using this $f\left(N_{B}\right)$, Algorithm 2 calculates the optimal number of BSs $N_{B}^{*}$ and corresponding minimized operational cost $f\left(N_{B}^{*}\right)$ by using golden section method that exploits the unimodality of operational cost $f\left(N_{B}\right)$ in $N_{B}$. The feasible search range of number of BSs $N_{B}$ lies between $N_{B}^{l}$ and $N_{B}^{u}$. We set the lower bound $N_{B}^{l}$ on $N_{B}$ as 1 and the upper bound $N_{B}^{u}$ as the maximum number $N_{B \text {, max }}$ of BSs that are available for deployment based on the overall budget. This search space $N_{B}^{u}-N_{B}^{l}$ reduces by a fixed factor of 0.618 after each iteration. The detailed steps followed in finding $N_{B}^{*}$ are mentioned in Algorithm 2. Since the objective is unimodal in $N_{B}$ and golden section algorithm is known to have fast convergence for unimodal functions [33], Algorithm 2 finds $N_{B}^{*}$ in very few iterations, which is described as follows: The algorithm stops after number of iterations $N_{\text {itr }}$ if $\left(N_{B}^{u}-N_{B}^{l}\right)(0.618)^{N_{\text {itr }}} \leq \varsigma$ which gives $N_{\text {itr }} \leq 2 \ln \left(\frac{N_{B}^{u}-N_{B}^{l}}{\varsigma}\right)$, where $\varsigma$ is the acceptable tolerance. In the performed experiments in Section VII, $N_{B}^{l}=1, N_{B}^{u}=35$, and acceptable threshold $\varsigma=1$. Hence, the proposed algorithms converge in $N_{\text {itr }}=7$ iterations which can be further reduced by smartly choosing the value of $N_{B}^{l}$ and $N_{B}^{u}$ depends on the channel conditions and coverage demand.

It can be observed that the optimization problem (P0) also maximizes delay-sensitive area spectral efficiency (DASE) [34, Section III] by providing a throughput at minimum affected area due to reduction in significant transmission power by the optimally deployed BSs. Further, the optimization is conducted offline because the redeployment of BSs incurs a monitory charge. Therefore before deployment of BSs, the optimal number of BSs $N_{B}^{*}$, their optimal locations $\mathbf{d}^{*}$ and optimal transmit power $P_{t}^{*}$ are evaluated in background for satisfying a coverage constraint. But using (9), it is possible to optimize $P_{t}$ online with constraint $\epsilon$ for a fixed deployment $\left(\mathbf{d}, N_{B}\right)$.

\section{The Effect of Interference on Cost Minimization}

Now, we conceive the changes in the optimization process, when the interference is considered in the framework. The optimization of location of BSs is based on the minimization of farthest UE's distance from the BS which is minimized by minimizing the farthest point Euclidean distance of the cell (cf. Section IV). As minimization of farthest point Euclidean distance is not affected by the interference over the cells, there is no change in optimization of location of BSs.

In the homogeneous network, the same optimal power $P_{t}^{*}$ is allocated to all BSs. It causes a sufficient enough signal is received by the farthest UE of larger cells, whereas a stronger signal is received in smaller cells. Therefore, the neighboring cells of smaller cells experience higher interference than the larger cells. In other words, the interference effect over the different cells are different depends on the size of its neighboring cells. The variation of the interference effect with respect to size of the cells motivates to allocate the adaptive power at the BSs for almost same interference effect over the network. The advantage of same interference effect is that it avoids the complication in the optimization process. If we allocate the power at the BSs adaptively such that nearly same signal strength reaches at the farthest UE of each cell to just satisfy the constraint, then the neighboring region of a cell face almost same interference effect irrespective of size of the cell. Thus, the interference effect over whole network is nearly same. For an average interference power $I$ at the farthest UE of each cell, the signal-to-interference-plus-noise ratio (SINR) at the farthest UE in $i^{\text {th }}$ cell is given as: $\mathrm{SINR}_{i}=$ $\frac{P_{i} h_{\mathrm{far}, i} r_{\mathrm{far}, i}^{-\alpha}}{I+\sigma^{2}}$. The corresponding average coverage probability can be expressed as: $P_{\mathrm{cov}}^{\mathrm{far}, i}=\mathbb{E}_{r_{\mathrm{far}, i}}\left\{\operatorname{Pr}\left[\frac{P_{i} h_{\mathrm{far}, i} r_{\mathrm{far}, i}^{-\alpha}}{I+\sigma^{2}} \geq T\right]\right\}=$ $\int_{0}^{r_{u, i}} \mathrm{e}^{-\frac{T\left(I+\sigma^{2}\right) r_{\mathrm{far}, i}^{\alpha}}{P_{i}}} f_{\mathrm{far}, i}\left(r_{\mathrm{far}, i}, \mathbf{d}_{\mathbf{i}}\right) \mathrm{d} r_{\mathrm{far}, i}, \quad \forall i$. Again, for a coverage probability $\geq 90 \%$, we get the allocated power $P_{i} \geq \frac{T\left(I+\sigma^{2}\right)}{\epsilon} \int_{0}^{r_{u, i}} r_{\mathrm{far}, i}^{\alpha} f_{\mathrm{far}, i}\left(r_{\mathrm{far}, i}, \mathbf{d}_{\mathbf{i}}^{*}\right) \mathrm{d} r_{\mathrm{far}, i}$ with a percentage error $\leq 0.053 \%$ as described in Section V-A. So, the optimal power over the $i^{t h}$ cell is given as: $P_{i}^{*}=$ $\frac{T\left(I+\sigma^{2}\right)}{\epsilon} \int_{0}^{r_{u, i}} r_{\mathrm{far}, i}^{\alpha} f_{\mathrm{far}, i}\left(r_{\mathrm{far}, i}, \mathbf{d}_{\mathbf{i}}^{*}\right) \mathrm{d} r_{\mathrm{far}, i}$. Here the interference power $I$ gives only a linear shift in computation of $P_{i}^{*}$.

The optimization of number of BSs $N_{B}$ is changed due to increase in number of variables in total cost due to adaptive power allocation which is given as: $\sum_{l=1}^{N_{B}}\left(a_{B} P_{l}+b_{B}\right)=$ $a_{B} \sum_{l=1}^{N_{B}} P_{l}+N_{B} b_{B}$. As the total cost is a function of $N_{B}$ variables $\left\{P_{l}\right\}_{l=1}^{N_{B}}$, it is complicated to find the unimodality of total cost in $N_{B}$ which requires an exhaustive study. This optimization in the presence of interference is out of scope of the current work due to space limitation.

\section{Cost Minimization in a SQuare Region}

As a square field is symmetric along its length and width, the cells are generated without any coverage hole by dividing the field along its length and width as shown in Fig. 5, where the length and width are divided into $p$ and $q$ equal segments, respectively, i.e., the number of cells $=p q=N_{B}$. The generated cells are square if $p=q$, otherwise they are rectangular. It is evident that rectangular and square cells are 


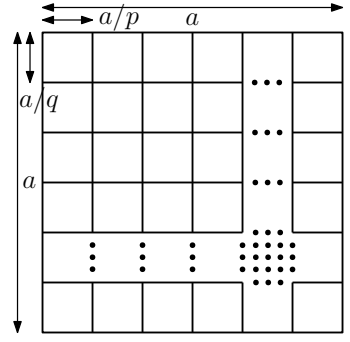

Fig. 5: Generation of cells over a square field of side length $a$.

equally divided by two orthogonal axis along the length and width with center as its origin, so the optimal location of the BSs is at the center of the cells $\forall N_{U}$ (cf. Section IV).

We aim to obtain the condition on $p$ and $q$ for finding the minimum value of farthest point Euclidean distance from the center. If we relax the integer value of $p$ and $q$, and set $q=$ $p-x(x \geq 0)$, then farthest point euclidean distance in a cell is $r_{u, c}=\frac{a}{2} \sqrt{\frac{1}{p^{2}}+\frac{1}{(p-x)^{2}}}$, where $a$ is side length of the square field. Minimum value of $r_{u, c}$ depends on the minimum value of $\mathcal{D}=\frac{1}{p^{2}}+\frac{1}{(p-x)^{2}}$. As $N_{B}=p(p-x) \Rightarrow p=\frac{x+\sqrt{x^{2}+4 N_{B}}}{2}$, $\mathcal{D}=\frac{x^{2}+2 N_{B}}{N_{B}^{2}}, \frac{\partial \mathcal{D}}{\partial x}=\frac{2 x}{N_{B}^{2}}, \frac{\partial^{2} \mathcal{D}}{\partial x^{2}}=\frac{2}{N_{B}^{2}}>0$, i.e., $r_{u, c}$ is convex and achieves a minimum value at $x^{*}=0$, i.e., at $p=q$. But for a given $N_{B}$, if $p=q$ is not possible for the integer value of $p$ and $q$, then $N_{B}=p q$ should be such that $x=|p-q|$ must be a minimum possible integer. Next, we discuss about the operational cost minimization in a square field.

\section{A. When the Density of UEs is Asymptotically Very High}

As discussed in Section IV, $f_{\mathrm{far}, c}\left(r_{\mathrm{far}, c}\right)=\delta\left(r_{\mathrm{far}, c}-r_{u, c}\right)$ for $N_{U} \rightarrow \infty$, where $f_{\mathrm{far}, c}\left(r_{\mathrm{far}, c}\right)$ is PDF of distance $r_{\mathrm{far}, c}$ of farthest UE in a cell of the square field. Here a cell is denoted by a suffix $c$, because all the cells are same. Also, we have dropped the location of the BS in the PDF expression as it is trivial that the BSs are lying at center of the cells. For coverage above $90 \%(\epsilon \leq 0.1)$, the tight bound of power allocation over the BSs is $P_{t} \geq \frac{T \sigma^{2}}{\epsilon} \int_{0}^{r_{u, c}} r_{\mathrm{far}, c}^{\alpha} f_{\mathrm{far}, c}\left(r_{\mathrm{far}, c}\right) \mathrm{d} r_{\mathrm{far}, c}=\frac{T \sigma^{2}}{\epsilon} r_{u, c}^{\alpha}$ as discoursed in Section V-A. For $N_{B}=p q, r_{u, c}=$ $\frac{a}{2} \sqrt{\frac{1}{p^{2}}+\frac{1}{q^{2}}}$; therefore, optimal power allocation in each cell is $P_{t}^{*}=\frac{T \sigma^{2}}{\epsilon}\left(\frac{a}{2} \sqrt{\frac{1}{p^{2}}+\frac{1}{q^{2}}}\right)^{\alpha}$. Now problem (P0) can be written as:

$$
\begin{aligned}
\text { (P1): } \underset{N_{B}>0}{\operatorname{minimize}} & N_{B}\left[c_{B}\left(\frac{a}{2} \sqrt{\frac{1}{p^{2}}+\frac{1}{q^{2}}}\right)^{\alpha}+b_{B}\right], \\
\text { subject to } & C 5: N_{B} \leq N_{B, \max }
\end{aligned}
$$

where $c_{B}=\frac{T \sigma^{2}}{\epsilon}$. The objective function of problem (P1) is unimodal and pseudoconvex with respect to $N_{B}$, which can be explained same as the discourse in Section V-B. One of the method for determining the minimized operational cost is by defining a function $f\left(N_{B}\right)$ like in Algorithm 1 in which first we find the optimal way of division of cells in the square field for a given value of $N_{B}$ for minimization of farthest point Euclidean distance, i.e., $N_{B}=p q$ such that $|p-q|$ is a minimum possible integer. Then we can find the $P_{t}^{*}=$ $\frac{T \sigma^{2}}{\epsilon}\left(\frac{a}{2} \sqrt{\frac{1}{p^{2}}+\frac{1}{q^{2}}}\right)^{\alpha}$ and corresponding operational cost as a output for a given value of $N_{B}$. Finally using the Algorithm 2, we can find $N_{B}^{*}$ and optimal operational cost. The drawback of this approach is that we have to apply Algorithm 2 over whole range of $N_{B} \in\left[1, N_{B \text {, max }}\right]$ In this case, we can make Algorithm 2 more efficient by reducing the range of $N_{B}$ by a very large factor for the iteration. First we take those $N_{B}$ values in which $N_{B}=p^{2}(p=q)$, then we find closed form solution of optimal number of BSs $N_{B, p^{2}}^{*}$. Lastly, we restrict the range of $N_{B}$ around $N_{B, p^{2}}^{*}$ for the iteratively determining $N_{B}^{*}$. For $N_{B}=p^{2}$, the problem $(\mathrm{P} 1)$ can be written as:

$$
\text { (P2) : } \underset{N_{B}>0}{\operatorname{minimize}} N_{B}\left[c_{B}\left(\frac{a}{\sqrt{2 N_{B}}}\right)^{\alpha}+b_{B}\right] \text {, subject to } C 5 \text {. }
$$

The objective function of (P1) is strictly convex because its second derivative $\frac{\alpha(\alpha-2) c_{B} a^{\alpha}}{N_{B}^{(\alpha / 2+1)} 2^{(\alpha / 2+2)}}>0$ for $\alpha>2$. The optimal solution is $N_{B, p^{2}}^{*}=\frac{a^{2}}{2}\left\{\frac{(\alpha / 2-1) c_{B}}{b_{B}}\right\}^{2 / \alpha}$. As $N_{B, p^{2}}^{*}$ may be a fractional value, the restricted range of $N_{B}$ for iterative solution is $\left[\left\lfloor\sqrt{N_{B, p^{2}}^{*}}\right\rfloor^{2},\left[\sqrt{N_{B, p^{2}}^{*}}\right]^{2}\right]$ for $P_{t, \max }<\frac{b_{B} C_{B}^{\alpha-1}}{\alpha / 2-1}$ and $\left[\left\lfloor\left.\sqrt{\frac{1}{2} \frac{\left(a C_{B}\right)^{2}}{\left(P_{t, \max }\right)^{2 / \alpha}}}\right|^{2},\left[\sqrt{\frac{1}{2} \frac{\left(a C_{B}\right)^{2}}{\left(P_{t, \text { max }}\right)^{2 / \alpha}}}\right]^{2}\right]\right.$ for $P_{t, \text { max }}>\frac{b_{B} C_{B}^{\alpha-1}}{\alpha / 2-1}$ For $\alpha=2$, the objective function is a affine transform of $N_{B}$ which gives $N_{B}^{*}=\max \left\{1,\left\lceil\frac{1}{2} \frac{\left(a C_{B}\right)^{2}}{P_{t, \max }}\right\rceil\right\}$.

\section{B. When the Density of UEs is Moderate}

Using (3), the PDF of distance of farthest UE in a cell is:

$$
\begin{aligned}
f_{\text {far }, c}\left(r_{\text {far }, c}\right)= & \sum_{z=0}^{N_{U}}\left(\begin{array}{c}
N_{U} \\
z
\end{array}\right)\left(\frac{1}{N_{B}}\right)^{z}\left(1-\frac{1}{N_{B}}\right)^{\left(N_{U}-z\right)} \\
& \times z f_{c}\left(r_{\mathrm{far}, c}\right)\left[F_{c}\left(r_{\mathrm{far}, c}\right)\right]^{(z-1)},
\end{aligned}
$$

where $f_{c}\left(r_{\mathrm{far}, c}\right)$ and $F_{c}\left(r_{\mathrm{far}, c}\right)$ are the PDF and CDF respectively of distance $r_{\mathrm{far}, c}$ of an UE in a cell. Corresponding optimal power allocation in every cell is $P_{t}^{*}=$ $\frac{T \sigma^{2}}{\epsilon} \int_{0}^{r_{u, c}} r_{\mathrm{far}, c}^{\alpha} f_{\mathrm{far}, c}\left(r_{\mathrm{far}, c}\right) \mathrm{d} r_{\mathrm{far}, c}$ for coverage above $90 \%$ (cf. Section V-A). Using $P_{t}^{*}$, the problem (P0) can be written as:

(P3) : $\underset{N_{B}}{\operatorname{minimize}} N_{B}\left[P_{t}^{*}+b_{B}\right], \quad$ subject to $C 3, C 5$.

Similar to discussion in Sections V-B and VI-A, the objective function of problem (P3) is unimodal and pseudoconvex with respect to $N_{B}$. Here also, first we take $p=q$, i.e., $r_{u, c}=\frac{a}{\sqrt{2 N_{B}}}$, then define $f\left(N_{B}\right)=$ $N_{B}\left[c_{B} \int_{0}^{\frac{a}{\sqrt{2 N_{B}}}} r_{\mathrm{far}, c}^{\alpha} f_{\mathrm{far}, c}\left(r_{\mathrm{far}, c}\right) \mathrm{d} r_{\mathrm{far}, c}+b_{B}\right]$. Using Algorithm 2, we find the optimal number of BSs $N_{B, p^{2}}^{*}$. Now, we can restrict the range of $N_{B}$ to $\left[\left(\sqrt{N_{B, p^{2}}}-1\right)^{2},\left(\sqrt{N_{B, p^{2}}}+\right.\right.$ $\left.1)^{2}\right]$. After redefining objective of $(\mathrm{P} 3)$ as $f\left(N_{B}\right)$, we can find the optimal number of BSs $N_{B}^{*}$ and minimized cost using Algorithm 2 in the restricted range of $N_{B}$.

If we compare problems (P1), (P2), and (P3) with the original problem $(\mathrm{P} 0)$, optimal location of the $\mathrm{BSs}$ is trivially located at the center of each cell from which their farthest point Euclidean distance is equal. The procedure of calculation of $P_{t}^{*}$ is same in all the optimization problems. But for $N_{B}^{*}$, (P0), (P1), and (P3) use the Algorithm 1 and Algorithms 2, whereas (P2) has a closed form expression due to simplicity in its cost function. Next, we will obtain the numerical results of proposed analytical system for minimization of the cost. 
TABLE II: System parameters with their default values.

\begin{tabular}{|c|c|c|}
\hline System parameter & Symbol & Value \\
\hline Radius of the circular field & $R$ & $500 \mathrm{~m}$ \\
\hline Side length of the square field & $a$ & $500 \sqrt{\pi} \mathrm{m}$ \\
\hline Number of UEs & $N_{U}$ & 120 \\
\hline Maximum number of deployed BSs & $N_{B, \max }$ & 35 \\
\hline Coefficient of power consumption & $a_{B}$ & $5.5[25]$ \\
\hline Additive power consumption & $b_{B}$ & $32 \mathrm{~W}[25]$ \\
\hline Maximum transmit power & $P_{t, \max }$ & $5 \mathrm{~W}[35$, part 95.135$]$ \\
\hline Acceptable threshold & $\epsilon$ & $10^{-2}$ \\
\hline SNR threshold & $T$ & $-10 \mathrm{~dB}$ \\
\hline Path loss exponent & $\alpha$ & 4 \\
\hline Noise power & $\sigma^{2}$ & $-70 \mathrm{dBm}$ \\
\hline Maximum density of BSs & $\lambda_{\max }$ & $5 \times 10^{-5} \mathrm{~m}^{-2}$ \\
\hline
\end{tabular}

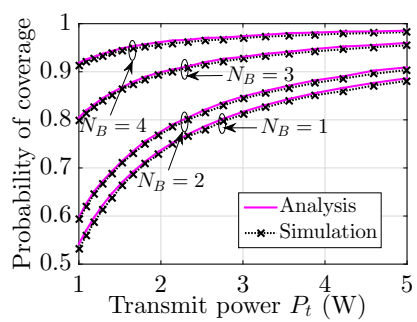

(a)

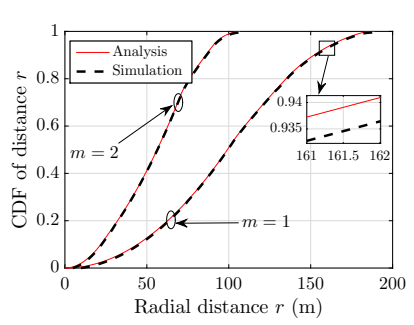

(b)
Fig. 6: Variation of average coverage probability and CDF of distance $r$ in $m^{t h}$ cell of $\mathcal{M}: m k+1(k=10)$ with $P_{t}$ and $r$ for different values of $N_{B}$ and $m$, respectively.

\section{NUMERICAL RESUlTS AND DISCUSSION}

Now we conduct numerical investigation on the proposed optimization and solution methodology. The system parameters and their default values have been listed in Table II. In fixed allocation scheme for experiments over the circular field, $P_{t}=4 \mathrm{~W}, N_{B}=35$, sectoring $\mathcal{M}: k(m=1, t=0)$, location $d_{1}=250 \mathrm{~m}$ for $N_{B} \geq 2 ; d_{1}=0$ for $N_{B}=1$.

\section{A. Validation of Analysis}

Firstly, we validate the average coverage probability expression given in (4). For validation, the simulation results are obtained by first examining $10^{6}$ random realizations of Rayleigh fading channel gain for the corresponding received SNR at the farthest UE in a cell to be greater than $-10 \mathrm{~dB}$ for a given UE deployment. After that the average of this fraction, for which SNR $\geq-10 \mathrm{~dB}$, is taken over the $10^{3}$ random UE deployments. A closed match between analytical and simulation as observed in Fig. 6(a), validates the average coverage probability analysis as discussed in Section III-A. We also verified the quality of approximation (8) for the average coverage probability constraint $C 1$ by noting that the corresponding root-mean-square error was less than 0.018 for $N_{B}=4$. As mentioned in Section V-A, this approximation error diminishes very rapidly with decreasing threshold $\epsilon$.

From Fig. 6(a), we also observe that there is not much improvement in the average coverage probability when two BSs are deployed instead of one BS. This is so because as center is optimal BS location in both cases, there is no reduction in the distance of the farthest point inside a cell from its BS on increasing $N_{B}$ from 1 to 2 . Through a high improvement in average coverage probability, when $N_{B}$ is

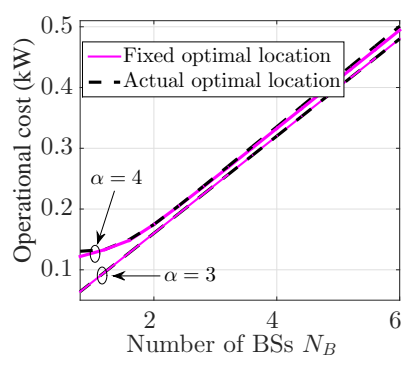

(a)

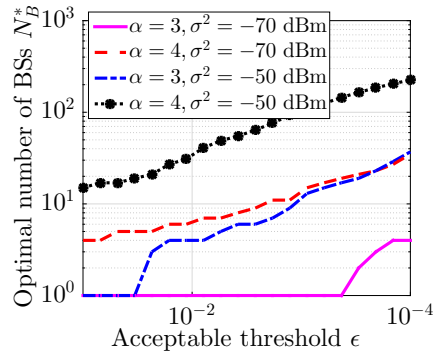

(b)
Fig. 7: (a) Difference in the operational costs of the BSs deployment scenarios. (b) Variation of optimal BSs count $N_{B}^{*}$ with $\epsilon, \alpha$ and $\sigma^{2}$.

increased from 2 to 3 , the improvement margin again decreases for $N_{B}=4$. So, we note that when the cells are generated by dividing the field in angular direction only, then the reduction rate of farthest point distance from the BS decreases with $N_{B}$. So, for higher improvement in reduction of the farthest point distance, we move to higher sectoring types, where the cells are generated in both angular and radial directions (cf. Fig. 1).

Also, we have validated the proposed CDF in appendix A of distance $r$ of an UE from the BS in $m^{\text {th }}$ cell for sectoring $\mathcal{M}: m k+1$ where $k=10$. It can be observed in Fig. 6(b) that the CDF reaches to value 1 at a faster rate for higher $m$ because the farthest point Euclidean distance from the BSs in each cell is reducing rapidly with $m$.

Lastly, we investigate the quality of approximation for the optimal BS location based on the ideology of minimizing the farthest point Euclidean distance in each cell. In this regards, in Fig. 7(a) we have plotted the cost performance for (a) BSs localization based on the minimization the farthest point Euclidean distance in each cell (called fixed optimal location) and (b) BSs localization based on the optimal locations (called actual optimal location) as found by searching in the neighborhood of the ones that minimize the farthest point Euclidean distance in each cell (cf. Section IV). As in Fig. 7(a), for both $\alpha=3$ and $\alpha=4$, the cost with fixed optimal location is in close match with the one for the optimal BS location. This validates our proposal and therefore, hereafter the deployment of BSs based on fixed optimal location has been taken in our experiments for simplicity.

\section{B. Role of Key System Parameters}

Now, we investigate the impact of channel conditions ( $\alpha$ and $\sigma^{2}$ ) on optimal number of BSs $N_{B}^{*}$ as obtained using the proposed joint optimization. As shown in Fig. 7(b), generally the increase in coverage demand, as represented by decreasing $\epsilon$, results in a significant increase in $N_{B}^{*}$. However, for $\alpha=3$ and $\sigma^{2}=-70 \mathrm{dBm}$ that represents the most favorable channel conditions, $N_{B}^{*}=1$ is sufficient for meeting high coverage quality demand with thresholds upto $\epsilon \geq 4.3 \times 10^{-4}$. For, $\alpha=3$, an increase in $\sigma^{2}$ from $-70 \mathrm{dBm}$ to $-50 \mathrm{dBm}$ results in an average increase of about 5.53 times in $N_{B}^{*}$. Similarly, when $\alpha$ increases from 3 to 4 for $\sigma^{2}=-70 \mathrm{dBm}, N_{B}^{*}$ on an average gets increased by 7.6 times. Thus, the optimal BSs 


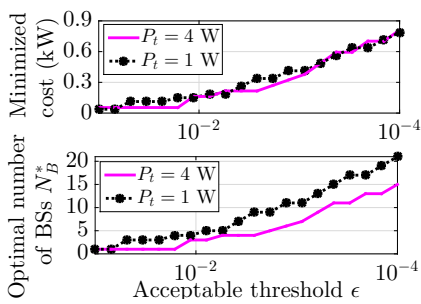

(a) Circular field

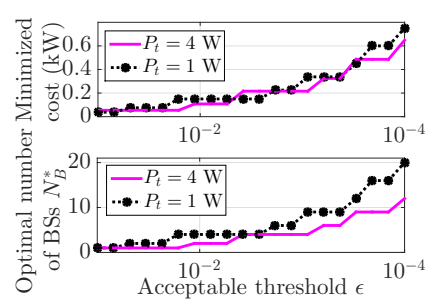

(b) Square field
Fig. 8: Variation of minimized cost and $N_{B}^{*}$ with $\epsilon$ and $P_{t}$.

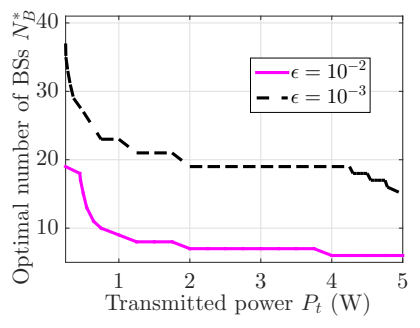

(a)

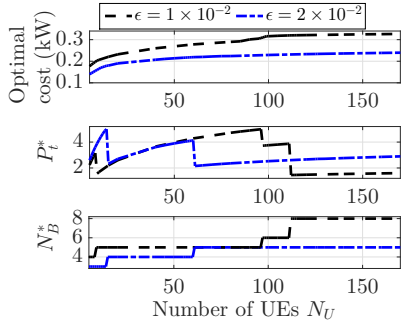

(b)
Fig. 9: Variation of optimal cost, $N_{B}^{*}, P_{t}^{*}$ for different $\epsilon$ and $N_{U}$.

count $N_{B}^{*}$ not only depends on the coverage threshold $\epsilon$, but is also strongly affected by channel conditions $\left(\alpha, \sigma^{2}\right)$.

In Fig. 8, we have plotted the tradeoff between the minimized operational cost and the underlying average coverage probability requirement $1-\epsilon$ in a circular and square field for $\sigma^{2}=-80 \mathrm{dBm}$. Here, $N_{B}$ and BS locations are jointly optimized for a given $P_{t}$ value. We notice that the optimal number of BSs $N_{B}^{*}$ is lower for higher $P_{t}$ and vice versa. This results in almost the same cost for the two $P_{t}$ values, because, for lower $P_{t}$, higher number of BSs are deployed and for higher $P_{t}, N_{B}^{*}$ is relatively lower. As acceptable average coverage probability increases from 0.9 to 0.9999 , the corresponding cost increasing from $40 \mathrm{~W}$ to $800 \mathrm{~W}$ and from $40 \mathrm{~W}$ to $700 \mathrm{~W}$ corroborates the utility of the proposed framework for $\epsilon \ll 1$ in the circular and square fields, respectively. It can also be observed in Fig. 8(a) that the required number of BSs $N_{B}$ configured at $P_{t}=1 \mathrm{~W}$ are 11 and 21 with average cell radius $156 \mathrm{~m}$ and $114 \mathrm{~m}$ for satisfying the coverage constraint $\epsilon=10^{-3}$ and $\epsilon=10^{-4}$, respectively, i.e., higher the coverage demand, larger number of BSs are deployed for satisfying the metric. Similarly, it can also be observed in the case of square field as shown in Fig. 8(b). Hence, as the coverage demand increases, a better link quality is required which is accomplished by reducing the cell radius. This reduction due to deployment of large number of BSs results in ultra-dense deployment of small cells [36].

The plots of optimal number of BSs $N_{B}^{*}$ with transmitted power $P_{t}$ for satisfying the thresholds $\epsilon=10^{-2}$ and $\epsilon=10^{-3}$ have been shown in Fig. 9(a). At $P_{t}=0.25 \mathrm{~W}$ and $P_{t}=5 \mathrm{~W}$, we require 18 and 9 more optimal number of BSs respectively for $\epsilon=10^{-3}$ than $\epsilon=10^{-2}$. Therefore, the requirement of more optimal number of BSs on average decreases with increment in $P_{t}$ and both the curves will asymptotically converge to $N_{B}^{*}=1$ for very high value of $P_{t}$. As shown in

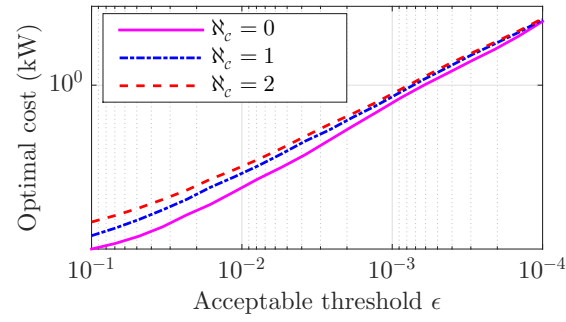

Fig. 10: Performance comparison for different $\aleph_{\mathcal{C}}$ values.

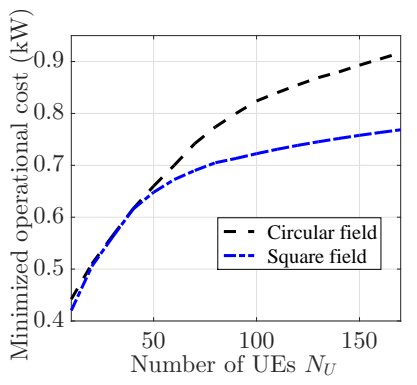

(a) Variation with number $N_{U}$ of UEs

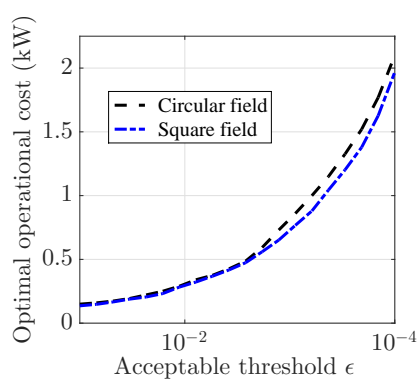

(b) Variation with threshold $\epsilon$
Fig. 11: Comparison of minimized cost in circular and square fields.

Fig. 9(b), the rate of increment in operational cost with number of UEs $N_{U}$ increases with increment in coverage demand. Also, when the number of optimal number of BSs $N_{B}^{*}$ is constant with increment in $N_{U}$, then the optimal transmitted power $P_{t}^{*}$ increases upto $P_{t, \max }=5 \mathrm{~W}$ and suddenly drops, when $N_{B}^{*}$ increases. Therefore, there is no sudden change in the minimized operational cost due to trade-off nature between $N_{B}^{*}$ and $P_{t}^{*}$ as discussed in Fig. 8.

Now we measure the degradation in the optimization technique in a practical scenario when some cells are not available for the deployment of BSs over the circular field. In Fig. 10, the degradations in the joint optimization scheme are evaluated for $\aleph_{c}=1,2$ and compared with the proposed work where $\aleph_{\mathcal{C}}=0$. Here $\aleph_{\mathcal{c}}$ denotes the number of randomly picked cells not available for the deployment of BSs. It can be observed from the plot that for low constraint $1-\epsilon$, the optimal number of BSs $N_{B}^{*}$ is quite less and even for $\aleph_{c}=1,2$, the optimal placement of other BSs is severely affected. Therefore, the performance degradation for low constraint is quite high as compared to the larger constraints, where $N_{B}^{*}$ is high and placement of other BSs is less affected. On average $13.15 \%$ and $21.41 \%$ more power cost take place in case of $\aleph_{c}=1$ and 2 respectively as compared to the optimization with $\aleph_{\mathcal{c}}=0$.

\section{Performance Comparison Results}

In Fig. 11, we compare the minimized operational cost in circular and square fields of same area. Initially, they follow each other with respect to $N_{U}$ and the difference gradually enhances after $N_{U}=40$ for satisfying the threshold $\epsilon=10^{-3}$ at $\sigma^{2}=-70 \mathrm{dBm}$ as shown in Fig. 11(a). The minimized operational cost in circular field is $12 \mathrm{~W}$ and $148.5 \mathrm{~W}$ higher than in square field for $N_{U}=50$ and $N_{U}=170$, respectively. But with respect to threshold $\epsilon$, this cost is almost same for 


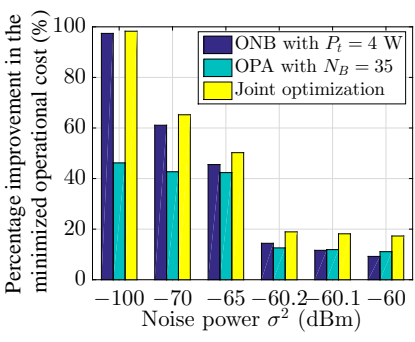

(a) Varying $\sigma^{2}$

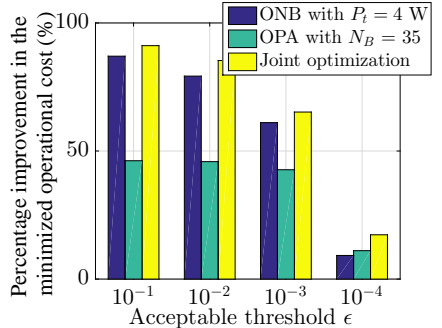

(b) Varying $\epsilon$
Fig. 12: Percentage improvement of different optimization schemes.

$\epsilon \geq 0.002$ otherwise minor increment in the cost occurs in circular field for $N_{U}=120$ as depicted in Fig. 11(b).

Finally, we conduct a comparison study in the circular field, where the relative performance of three optimization schemes, (i) ONB: optimal number of BSs for $P_{t}=4 \mathrm{~W}$, (ii) OPA: optimal power allocation for $N_{B}=35$, and (iii) proposed joint optimization, are compared against the fixed allocation scheme. The optimization with respect to the BS locations and sectoring type is considered for all three schemes under different noise power $\sigma^{2}$ and acceptable threshold $\epsilon$ settings. From Figs. 12(a) and 12(b), we note that ONB has better performance than OPA for $\sigma^{2} \leq-60.1 \mathrm{dBm}$ and $\epsilon=\left\{10^{-1}\right.$, $\left.10^{-2}, 10^{-3}\right\}$, respectively. However, for very high noise power $\sigma^{2} \geq-60 \mathrm{dBm}$ or for very high coverage demand with $\epsilon=$ $10^{-4}$, a large number of BSs are needed to be deployed. This happens because as OPA is already having very high $N_{B}=35$, which is near optimal for $\sigma^{2} \geq-60 \mathrm{dBm}$ and $\epsilon=10^{-4}$, the optimization with respect to $P_{t}$ helps OPA in achieving better performance than ONB for higher noise power scenarios or higher QoS applications (lower $\epsilon$ ). The best performance is achieved by the proposed joint optimization scheme, which yields an average reduction of about $65 \%$ in the operational cost with varying QoS or coverage demands (represented by varying $\epsilon$ ) as compared to the fixed allocation scheme.

Now we compare our proposed joint optimization algorithm with the optimization techniques given in [24] and [25] in a noise limited homogeneous network. In both the existing algorithms, the objective function area power consumption (APC) is confined to the area of the circular field and average coverage probability of an UE is considered as a constraint. Also, number of antennas $M=1$ is taken in [24] for reasonable comparison of its algorithm with other schemes. If we compare the optimization methods, the proposed optimization is better than the existing works as shown in Fig. 13. On average the proposed optimization requires nearly $39 \%$ and $48 \%$ lesser operational cost than the existing works with respect to $\epsilon$ and $\sigma^{2}$, respectively. This gives an insight that the deterministic optimal deployment of BSs has a significant role in minimizing the operational cost in high coverage demand and noisy channel. Here the existing works [24] and [25] almost give same performance in terms of operational cost minimization. This cost calculated in $\mathrm{kW}$, can also be expressed as monetary charges in US dollars (USD) per hour using an appropriate scale factor. For example, the monetary charges for the optimized operational cost as plotted

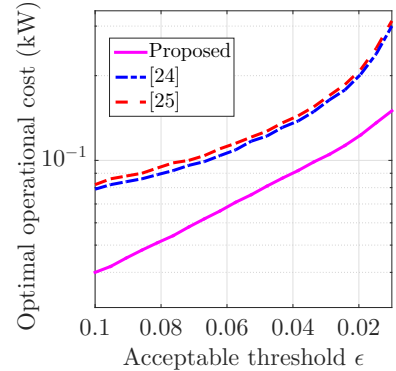

(a)

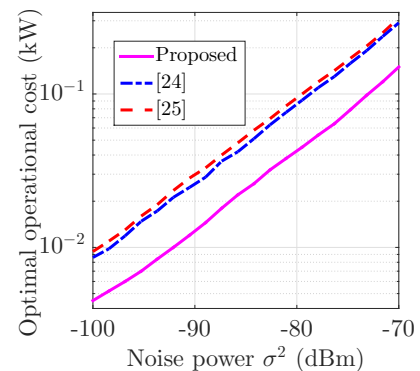

(b)
Fig. 13: Performance comparison of proposed scheme against ones in [24] and [25] for different (a) threshold $\epsilon$ and (b) noise power $\sigma^{2}$.

in Fig. 8(a) can be obtained by scaling it with an appropriate electricity price rate of say $0.0464 \mathrm{USD} / \mathrm{kWh}[37$, Section V].

\section{CONCLUding Remarks}

We have efficiently solved the non-convex combinatorial operational cost minimization problem by using a novel solution methodology that involves decoupling of the joint practical problem into three individual optimization problems. Firstly, insights on optimal BS location and sectoring type were provided. A tight approximation for transmit power allocation was presented for high coverage demands with $\epsilon \leq 0.1$. Lastly, the optimal number of BSs was found iteratively by exploiting the global-optimality in $N_{B}$. Later, we have extended the methodology in a square field for finding the minimized operational cost. Numerical results presented insights on the impact of various system parameters on the tradeoff between the optimized cost and coverage quality. It is observed that the proposed joint optimization framework, yielding a significant performance enhancement over the benchmark schemes, can help in the practical realization of green QoS-aware network operation. Also, a square field has better performance than a circular field in minimization of the operational cost.

The work performs the optimization in a SISO communication system over a $2 \mathrm{D}$ field. Along with the interference effect as discussed in Section V-C, the framework can also be carried out by comprising multiple-input and single-output (MISO) or 3D architecture [38] as a future work with following changes. There is no modification in optimization of location of BSs as it is based on minimization of farthest point Euclidean distance. But, the changes are incorporated with optimization of power allocation in the BSs on account of the precoding design in MISO or coverage probability in 3D model. In case of 3D system, the unimodality of total cost in $N_{B}$ can be shown similar to Section V-B for obtaining $N_{B}^{*}$. Whereas the complication arises in MISO communication system due to additional factors introduced in the total cost which depend on number of UEs lying over a cell and the precoding design. Further, the system model can be made more realistic by considering blockage effect as a function of distance [39, Section III] which can be pursued as a future direction. 


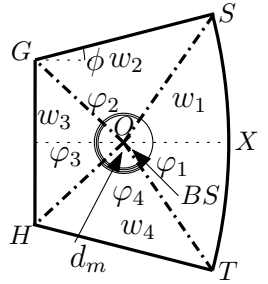

(a)

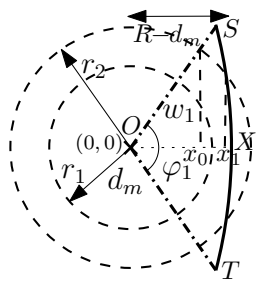

(b)
Fig. A.1: (a) Division of the $m^{t h}$ cell in four disjoint sub-fields $\left\{w_{j}\right\} ; j \in\{1,2,3,4\}$, (b) computation of distance distribution of an UE from location $d_{m}$ of the BS over the sub-field $w_{1}$.

\section{APPENDIX A \\ Distribution for Circular Arc Cell Boundary}

The possible shape of the cells generated by optimally deploying multiple BSs in a circular field are polygons except $m^{t h}$ cell in which one of the boundary is an arc of the circular field as shown in Fig. 1. Calculation of distance distribution in polygon shaped fields has been discussed in [31], where all the boundaries are straight-line segment. But, no discussion has been done in fields associated with a circular arc shaped boundary. Here, we have extended the procedure in [31] for calculation of distance distribution in $m^{\text {th }}$ cell.

Similar to [31], we have divided the $m^{\text {th }}$ cell field $W_{m}$ in four disjoint sub-fields $\left\{w_{j}\right\} ; j \in\{1,2,3,4\}$ about the location $d_{m}$ of the BS associated with each boundaries as shown in Fig. A.1(a), i.e. $W_{m}=w_{1} \cup w_{2} \cup w_{3} \cup w_{4}$. The distance distribution of an UE from the location $d_{m}$ i.e., the CDF $\frac{A_{j}(r)}{\operatorname{Ar}\left(W_{m}\right)}$ and PDF $\frac{\partial A_{j} / \partial r}{\operatorname{Ar}\left(W_{m}\right)} ; j \in\{1,2,3,4\}$ [40] in each sub-field is computed individually, where $\operatorname{Ar}\left(W_{m}\right)$ is the area of the field $W_{m}$ and $A_{j}(r)=\operatorname{Ar}\left(C\left(d_{m}, r\right) \cap w_{j}\right)$ is the area of intersection between the two fields: a bounded circle $C\left(d_{m}, r\right)$ with radius $r$ centered at $d_{m}$ and sub-field $w_{j}$. The overall distance distribution $F_{m}\left(r, \mathbf{d}_{\mathbf{m}}\right)$ over the $m^{t h}$ cell field $W_{m}$ as obtained by from those over the four sub-fields is given by:

$$
F_{m}\left(r, \mathbf{d}_{\mathbf{m}}\right)=\frac{1}{\operatorname{Ar}\left(W_{m}\right)} \sum_{j=1}^{4} A_{j}(r)
$$

where $\mathbf{d}_{\mathbf{m}}=\left\{d_{m-1}, d_{m}\right\}$ is the location of BSs in $(m-1)^{t h}$ and $m^{t h}$ cells, respectively. $F_{m}\left(r, \mathbf{d}_{\mathbf{m}}\right)$ is the CDF respectively of distance $r$ of an UE from the location $d_{m}$ of the BS. As the sub-fields $w_{2}, w_{3}$, and $w_{4}$ are associated with straightline segment shaped boundaries, the computation of distance distribution is same as discussed in [31]. Here we discuss the computation of distance distribution in the sub-field $w_{1}$ associated with a circular arc shaped boundary in a especial scenario, when the BS lie at the symmetric axis of the cell.

For more discernment, we have explored the sub-field $w_{1}$ in Fig. A.1(b). As the BS always reside on the symmetric line of the cell, the sub-field $w_{1}$ is symmetric about the axis $O X$. The bounded circle $C\left(d_{m}, r\right)$ interact with the boundaries in two discrete ranges $r_{1} \in\left[0, R-d_{m}\right)$ and $r_{2} \in\left[R-d_{m}, \sqrt{R^{2}+d_{m}^{2}-2 R d_{m} \cos \phi}\right]$. Using trigonomet- ric relationship, the area of the intersection field $A_{1}(r)=$ $\operatorname{Ar}\left(C\left(d_{m}, r\right) \cap w_{1}\right)$ can be computed as:

$A_{1}(r)=\left\{\begin{array}{l}\frac{\varphi_{1} r^{2}}{2} ; \text { for } 0 \leq r<R-d_{m} \\ \frac{\pi R^{2}}{2}+m x_{0}^{2}+x_{1} \sqrt{r^{2}-x_{1}^{2}}-x_{0} \sqrt{r^{2}-x_{0}^{2}} \\ -\left(x_{1}+d_{m}\right) \sqrt{R^{2}-\left(x_{1}+d_{m}\right)^{2}}+r^{2} \arctan \frac{x_{1}}{r^{2}-x_{1}^{2}} \\ -r^{2} \arctan \frac{x_{0}}{r^{2}-x_{0}^{2}}-R^{2} \arctan \frac{x_{1}+d_{m}}{\sqrt{R^{2}-\left(x_{1}+d_{m}\right)^{2}}} \\ \text { for } \quad R-d_{m} \leq r \leq \sqrt{R^{2}+d_{m}^{2}-2 R d_{m} \cos \phi}\end{array}\right.$

with its first derivative with respect to $r$ being expressed as:

$$
\frac{\partial A_{1}(r)}{\partial r}=\left\{\begin{array}{l}
\varphi_{1} r ; \text { for } 0 \leq r<R-d_{m} \\
\left(\varphi_{1}+2 \arctan \frac{x_{1}}{\sqrt{r^{2}-x_{1}^{2}}}-\pi\right) r ; \text { for } R-d_{m} \leq \\
r \leq \sqrt{R^{2}+d_{m}^{2}-2 R d_{m} \cos \phi},
\end{array}\right.
$$

with $\varphi_{1}=2\left\{\pi-\arccos \left(\frac{d_{m}-R \cos \phi}{\sqrt{R^{2}+d_{m}^{2}-2 d_{m} R \cos \phi}}\right)\right\}, m=\tan \left(\frac{\varphi_{1}}{2}\right)$, $x_{0}=\frac{r}{\sqrt{m^{2}+1}}, x_{1}=\frac{R^{2}-r^{2}-d_{m}^{2}}{2 d_{m}}$ are intersection points as shown in Fig. A.1(b), and $2 \phi$ is the angle of the sector in which $m^{t h}$ cell is one of the generated cell. Area of the field $W_{m}$ can be easily obtained as: $\boldsymbol{A r}\left(W_{m}\right)=\phi R^{2}-\frac{1}{4} \tan \phi\left(d_{m}+d_{m-1}\right)^{2}$, where $d_{m-1}$ is the location of $\mathrm{BS}$ in adjacent $(m-1)^{t h}$ cell. Now, after substituting (A.2) and (A.3) into (A.1), we obtain the desired CDF $F_{m}\left(r, \mathbf{d}_{\mathbf{m}}\right)$ and PDF $f_{m}\left(r, \mathbf{d}_{\mathbf{m}}\right)=$ $\frac{\partial F_{m}\left(r, \mathbf{d}_{\mathbf{m}}\right)}{\partial r}$. Note that in sectoring $k$, where $m=1$, the field $W_{m}$ has three boundaries (two straight-line segment and one circular arc), which generates three disjoint sub-fields.

\section{REFERENCES}

[1] G. Prasad, D. Mishra, and A. Hossain, "Coverage-constrained base station deployment and power allocation for operational cost minimization,' in Proc. IEEE PIMRC, Montreal, Canada, Oct. 2017, pp. 1-5.

[2] H. ElSawy, E. Hossain, and M. Haenggi, "Stochastic geometry for modeling, analysis, and design of multi-tier and cognitive cellular wireless networks: A survey," IEEE Commun. Surveys Tutorials, vol. 15, no. 3, pp. 996-1019, Jun. 2013.

[3] J. G. Andrews, F. Baccelli, and R. K. Ganti, "A tractable approach to coverage and rate in cellular networks," IEEE Trans. on Commun., vol. 59, no. 11, pp. 3122-3134, Nov. 2011.

[4] S. Srinivasa and M. Haenggi, "Distance distributions in finite uniformly random networks: Theory and applications," IEEE Trans. Veh. Technol., vol. 59, no. 2, pp. 940-949, Feb 2010.

[5] G. Su, L. Li, X. Lin, and H. Wang, "On the optimal small cell deployment for energy-efficient heterogeneous cellular networks," in Proc. IEEE Int. Conf. on Ubiquitous and Future Networks, Shanghai, China, Jul. 2014, pp. 172-175.

[6] G. Fettweis and E. Zimmermann, "ICT energy consumption-trends and challenges," in Proc. IEEE Int. Symp. Wireless Personal Multimedia Commun., Lapland, Finland, Sept. 2008, pp. 1-6.

[7] M. A. Marsan, L. Chiaraviglio, D. Ciullo, and M. Meo, "Optimal energy savings in cellular access networks," in Proc. IEEE ICC Workshops, Dresden, Germany, Jun. 2009, pp. 1-5.

[8] S. Bhaumik, G. Narlikar, S. Chattopadhyay, and S. Kanugovi, "Breathe to stay cool: adjusting cell sizes to reduce energy consumption," in Proc. of the First ACM SIGCOMM Workshop on Green Networking, New York, USA, Aug. 2010, pp. 41-46.

[9] E. Oh and B. Krishnamachari, "Energy savings through dynamic base station switching in cellular wireless access networks," in Proc. IEEE GLOBECOM, Miami, FL, USA, Dec. 2010, pp. 1-5.

[10] E. Oh, K. Son, and B. Krishnamachari, "Dynamic base station switchingon/off strategies for green cellular networks," IEEE Trans. on Wireless Commun., vol. 12, no. 5, pp. 2126-2136, Mar. 2013.

[11] J. Peng, H. Tang, P. Hong, and K. Xue, "Stochastic geometry analysis of energy efficiency in heterogeneous network with sleep control," IEEE Wireless Commun. Lett., vol. 2, no. 6, pp. 615-618, Dec. 2013. 
[12] S. Zhou, J. Gong, Z. Yang, Z. Niu, and P. Yang, "Green mobile access network with dynamic base station energy saving," in Proc. ACM MobiCom, Beijing, China, Sept. 2009, pp. 10-12.

[13] G. Jie, Z. Sheng, and N. Zhisheng, "A dynamic programming approach for base station sleeping in cellular networks," IEICE Trans. on Commun., vol. 95, no. 2, pp. 551-562, Feb. 2012.

[14] J. Wu, S. Zhou, and Z. Niu, "Traffic-aware base station sleeping control and power matching for energy-delay tradeoffs in green cellular networks," IEEE Trans. on Wireless Commun., vol. 12, no. 8, pp. 41964209, Aug. 2013.

[15] J. Wu, Y. Zhang, M. Zukerman, and E. K. N. Yung, "Energy-efficient base-stations sleep-mode techniques in green cellular networks: A survey," IEEE Commun. Surveys Tutorials, vol. 17, no. 2, pp. 803-826, Feb. 2015.

[16] W. Chen, H. Li, Z. Li, Z. Xiao, and D. Wang, "Optimization of small cell deployment in heterogeneous wireless networks," in Proc. IEEE Int. Conf. on Comput., Inform. and Telecommun. Syst., China, 2016, pp. 1-5.

[17] Y. Yang, L. Chen, W. Dong, and W. Wang, "Active base station set optimization for minimal energy consumption in green cellular networks," IEEE Trans. Veh. Technol., vol. 64, no. 11, pp. 5340-5349, Nov. 2015

[18] F. Richter, A. J. Fehske, and G. P. Fettweis, "Energy efficiency aspects of base station deployment strategies for cellular networks," in Proc. IEEE VTC Fall, Anchorage, AK, USA, Sept. 2009, pp. 1-5.

[19] D. Cao, S. Zhou, and Z. Niu, "Optimal combination of base station densities for energy-efficient two-tier heterogeneous cellular networks," IEEE Trans. Wireless Commun., vol. 12, no. 9, pp. 4350-4362, 2013.

[20] B. Perabathini, M. Kountouris, M. Debbah, and A. Conte, "Optimal area power efficiency in cellular networks," in Proc. IEEE GLOBECOM Workshops, Austin, TX, USA, Dec. 2014, pp. 1157-1161.

[21] S. Sarkar, R. K. Ganti, and M. Haenggi, "Optimal base station density for power efficiency in cellular networks," in Proc. IEEE ICC, Sydney, NSW, Australia, Jun. 2014, pp. 4054-4059.

[22] J. Yu, Y. Liu, and C. Yin, "Energy-efficient base station deployment in heterogeneous cellular network with qos constraint," in Proc. IEEE PIMRC Workshops, London, UK, Sept. 2013, pp. 62-30.

[23] E. Mugume, D. K. C. So, and E. Alsusa, "Energy efficient deployment of dense heterogeneous cellular networks," in Proc. IEEE GLOBECOM, San Diego, CA, USA, Dec. 2015, pp. 1-6.

[24] D. Verenzuela, E. Bjrnson, and L. Sanguinetti, "Optimal design of wireless networks for broadband access with minimum power consumption," in Proc. IEEE ICC, Kuala Lumpur, Malaysia, May 2016, pp. 1-6.

[25] J. Peng, P. Hong, and K. Xue, "Energy-aware cellular deployment strategy under coverage performance constraints," IEEE Trans. Wireless Commun., vol. 14, no. 1, pp. 69-80, Jan. 2015.

[26] P. Gonzalez-Brevis, J. Gondzio, Y. Fan, H. V. Poor, J. Thompson, I. Krikidis, and P. J. Chung, "Base station location optimization for minimal energy consumption in wireless networks," in Proc. IEEE VTC Spring, Yokohama, Japan, May 2011, pp. 1-5.

[27] J. G. Andrews, "Interference cancellation for cellular systems: a contemporary overview," IEEE Wireless Commun., vol. 12, no. 2, pp. 19-29, Apr. 2005.

[28] C. H. Liu and L. C. Wang, "Random cell association and void probability in poisson-distributed cellular networks," in Proc. IEEE ICC, London, UK, Jun. 2015, pp. 2816-2821.

[29] P. V. Mekikis, E. Kartsakli, A. S. Lalos, A. Antonopoulos, L. Alonso, and C. Verikoukis, "Connectivity of large-scale wsns in fading environments under different routing mechanisms," in Proc. IEEE ICC, London, UK, Jun. 2015, pp. 6553-6558.

[30] O. Arnold, F. Richter, G. Fettweis, and O. Blume, "Power consumption modeling of different base station types in heterogeneous cellular networks," in Proc. Future Network and Mobile Summit, Florence, Italy, Jun. 2010, pp. 1-8.

[31] B. K. Baltzis, "Distance distribution in convex n-gons: Mathematical framework and wireless networking applications," Wireless Personal Commun., Springer, no. 2, pp. 1487-1503, Jul. 2013.

[32] M. Avriel, E. Diewerth, S. Schaible, and I. Zang, Generalized concavity. Philadelphia, PA, USA: SIAM, vol. 63, 2010.

[33] A. D. Belegundu and T. R. Chandrupatla, Optimization concepts and applications in engineering. Cambridge University Press, 2011.

[34] B. Makki, C. Fang, T. Svensson, M. Nasiri-Kenari, and M. Zorzi, "Delay-sensitive area spectral efficiency: A performance metric for delay-constrained green networks," IEEE Trans. on Commun., no. 6, pp. 2467-2480, Jun. 2017.

[35] C. of Federal Regulation, Title 47. Washington, DC, USA: Govt Printing Office (revised), October 2008.
[36] X. Ge, S. Tu, G. Mao, C. X. Wang, and T. Han, "5G ultra-dense cellular networks," IEEE Wireless Commun., vol. 23, no. 1, pp. 72-79, Feb. 2016.

[37] S. Bu, F. R. Yu, Y. Cai, and X. P. Liu, "When the smart grid meets energy-efficient communications: Green wireless cellular networks powered by the smart grid," IEEE Trans. on Wireless Commun., vol. 11, no. 8, pp. 3014-3024, Aug. 2012.

[38] Z. Pan and Q. Zhu, "Modeling and analysis of coverage in 3-D cellular networks," IEEE Commun. Lett., vol. 19, no. 5, pp. 831-834, May 2015.

[39] T. Bai, R. Vaze, and R. W. Heath, "Analysis of blockage effects on urban cellular networks," IEEE Trans. on Wireless Commun., vol. 13, no. 9, pp. 5070-5083, Sept. 2014.

[40] D. Moltchanov, "Distance distributions in random networks," Ad Hoc Networks, Elsevier, vol. 10, no. 6, pp. 1146-1166, Aug. 2012.

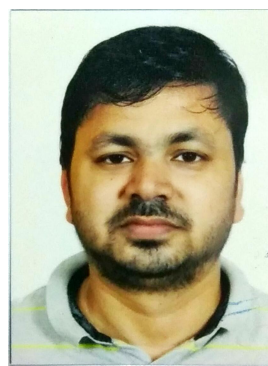

Ganesh Prasad (M'15) received the M. Tech degree in Electronics and Communication Engineering from Indian Institute of Technology (BHU), Varanasi, India, in 2012, and pursuing the Ph.D. degree in the same domain from National Institute of Technology (NIT), Silchar, India. He is currently an Assistant Professor in the Department of Electronics and Communication Engineering, NIT, Silchar. His research interests include cooperative wireless communications, underwater networks, free space optical communications, and green communications.

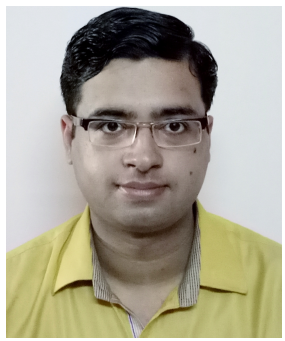

Deepak Mishra (S'13-M'17) received the B.Tech degree in Electronics and Communication Engineering from the Guru Gobind Singh Indraprastha University, Delhi, India, in 2012, and the Ph.D. degree in Electrical Engineering from the Indian Institute of Technology Delhi, India, in 2017. He is currently a Postdoctoral Researcher in the Department of Electrical Engineering (ISY), Linköping University, Linköping, Sweden. His research interests include energy harvesting cooperative communication networks, massive MIMO, physical layer security, signal processing and energy optimization schemes for uninterrupted operation of wireless networks. Dr. Mishra was selected as an Exemplary Reviewer of the IEEE TRANSACTIONS ON WIRELESS COMMUNICATIONS for 2017. He was a recipient of IBM Ph.D. Fellowship, Raman-Charpak Fellowship, and Endeavour Research Fellowship awards in 2016, 2017, and 2018, respectively.

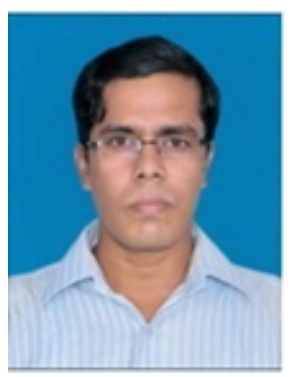

Ashraf Hossain (S'07-M'13-SM-17) received the B.Tech. and M.Tech. degrees in Radio Physics and Electronics from the Institute of Radio Physics and Electronics, University of Calcutta, Kolkata, India in 2002 and 2004, respectively. He earned the Ph.D degree in Electronics and Electrical Communication Engg., from Indian Institute of Technology (IIT), Kharagpur, India in 2011. He is currently working as an Assistant Professor in the Dept. of Electronics and Communication Engg., National Institute of Technology (NIT), Silchar, Assam, India. He served Dept. of Electronics and Communication Engg., Aliah University, Kolkata and Haldia Institute of Technology as Asst. Professor. His research interests include wireless sensor network, communication theory and systems, wireless network, cognitive radio. He is the Member of IE (India) 